\title{
Wilms' tumor 1 drives fibroproliferation and myofibroblast transformation in severe fibrotic lung disease
}

\author{
Vishwaraj Sontake, ${ }^{1,6}$ Rajesh K. Kasam, ${ }^{1,2,6}$ Debora Sinner, ${ }^{3,6}$ Thomas R. Korfhagen, ${ }^{3,6}$ \\ Geereddy B. Reddy, ${ }^{2}$ Eric S. White, ${ }^{4}$ Anil G. Jegga, ${ }^{5,6}$ and Satish K. Madala ${ }^{1,6}$ \\ 'Division of Pulmonary Medicine, Cincinnati Children's Hospital Medical Center, Cincinnati, Ohio, USA. Department of \\ Biochemistry, National Institute of Nutrition, Hyderabad, Telangana, India. ${ }^{3}$ Division of Neonatology and Pulmonary \\ Biology, Cincinnati Children's Hospital Medical Center, Cincinnati, Ohio, USA. ${ }^{4}$ Department of Internal Medicine, \\ University of Michigan Health System, Ann Arbor, Michigan, USA. ${ }^{5}$ Division of Biomedical Informatics Cincinnati \\ Children's Hospital Medical Center, Cincinnati, Ohio, USA. 'Department of Pediatrics, College of Medicine, University of \\ Cincinnati, Cincinnati, Ohio, USA.
}

Wilms' tumor 1 (WT1) is a critical transcriptional regulator of mesothelial cells during lung development but is downregulated in postnatal stages and adult lungs. We recently showed that WT1 is upregulated in both mesothelial cells and mesenchymal cells in the pathogenesis of idiopathic pulmonary fibrosis (IPF), a fatal fibrotic lung disease. Although WT1-positive cell accumulation leading to severe fibrotic lung disease has been studied, the role of WT1 in fibroblast activation and pulmonary fibrosis remains elusive. Here, we show that WT1 functions as a positive regulator of fibroblast activation, including fibroproliferation, myofibroblast transformation, and extracellular matrix (ECM) production. Chromatin immunoprecipitation experiments indicate that WT1 binds directly to the promoter DNA sequence of $\alpha$-smooth muscle actin ( $\alpha$ SMA) to induce myofibroblast transformation. In support, the genetic lineage tracing identifies WT1 as a key driver of mesothelial-to-myofibroblast and fibroblast-to-myofibroblast transformation. Importantly, the partial loss of WT1 was sufficient to attenuate myofibroblast accumulation and pulmonary fibrosis in vivo. Further, our coculture studies show that WT1 upregulation leads to non-cell autonomous effects on neighboring cells. Thus, our data uncovered a pathogenic role of WT1 in IPF by promoting fibroblast activation in the peripheral areas of the lung and as a target for therapeutic intervention.

Conflict of interest: The authors have declared that no conflict of interest exists.

Submitted: March 21, 2018

Accepted: July 11, 2018

Published: August 23, 2018

Reference information: JCI Insight. 2018;3(16):e121252. https://doi.org/10.1172/jci. insight.121252.

\section{Introduction}

Idiopathic pulmonary fibrosis (IPF) is a severe and chronic fibrotic lung condition that has been shown to associate with aging and is characterized by unrestrained mesenchymal cell activation such as proliferation, migration, differentiation, and extracellular matrix (ECM) deposition $(1,2)$. The prevalence of IPF is estimated to be between 1.25 and 23.4 cases per 100,000 , with IPF incidence at approximately 200,000 in the US alone $(3,4)$. Histological evaluations of IPF biopsies has revealed the pattern of pulmonary fibrosis that is predominant in subpleural and paraseptal areas with architectural distortion, such as honeycombing, septal thickening, and fibrotic foci in the lung parenchyma $(5,6)$. Recent pulmonary imaging studies suggest that the early fibrotic lesions are initiated in the subpleural areas of IPF lungs (7, 8). However, cellular and molecular mechanisms of mesenchymal cell activation in the formation of early lesions and progressive expansion in the peripheral areas of IPF lungs remain unknown. In IPF, lung mesenchymal cell genes are transcriptionally dysregulated, leading to uncontrolled profibrotic signaling; this ultimately results in myofibroblast accumulation and ECM deposition, causing impaired lung function. Unfortunately, there are no effective treatments to slow the rate of progression or reverse pathological alterations in IPF. The present study was undertaken with the goal of identifying transcription factors activating profibrotic gene networks in mesenchymal cells in the pathogenesis of IPF. Such transcription factors and gene networks may be targets for modulating the fibrotic processes leading to IPF.

Wilms' tumor 1 (WT1) is a zinc-finger transcription regulator that is shown to play a critical role in Wilms' tumor and hematological malignancies but is not previously known to have a role in the patho- 
genesis of pulmonary fibrosis (9-11). Previous studies have demonstrated the ability of WT1 to directly regulate gene expression and RNA metabolism involved in the formation, maintenance, and repair of several vital organs such as lung, heart, and kidney (12-18). WT1 is shown to play an oncogenic role in leukemic blast cells by increasing the proliferation and survival of progenitor cells $(9,11)$. A considerable body of evidence exists that suggests that WT1 can function as either a positive or negative regulator of proliferation by associating with crucial proteins involved in the checkpoint signaling in multiple cancers (19-21). In embryonic development of the lung, WT1 is selectively expressed by the majority of mesothelial cells lining the lung parenchyma, and its expression is critical for lung development (13). Importantly, the loss of the WT1 gene results in embryonic lethality in mice at E13.5-E14.5 (16). Further, WT1-positive cells of the embryonic lung have been shown to act as progenitors for fibroblasts and smooth muscle cells in perivascular and peribronchial areas of the adult lung $(13,14)$. After birth, the WT1 expression is downregulated, with limited or no expression in mesothelial cells of the lung. However, our recent study demonstrated that WT1 is upregulated in both mesothelial and mesenchymal cells at the early stages of fibrotic lung disease and remains upregulated in myofibroblasts that accumulate in the subpleural areas of fibrotic lungs (22). Still, the molecular and functional contribution of this potentially novel zinc finger transcription factor in fibroblast activation and the pathogenesis of IPF is unknown. A recent study suggests that WT1 is expressed in mesothelial cells of normal lungs, while it is decreased in IPF lungs (23). The authors that WT1 functions as a negative regulator of mesothelial-to-myofibroblast differentiation in IPF. In contrast, we and others observed limited or no staining for WT1 in normal adult human lungs but elevated staining in lung mesenchymal and mesothelial cells of human $\operatorname{IPF}(8,22,24)$. Further, our coimmunostaining studies showed that the reactivated WT1 is selectively localized in the nucleus of lung mesenchymal cells positive for either vimentin or $\alpha$-smooth muscle actin ( $\alpha$ SMA) in IPF compared with normal lungs. Therefore, it is essential to understand the mechanisms underpinning the detrimental impact of WT1 upregulation on fibroblast gene expression, which is critical for designing strategies to attenuate IPF and other fibrotic lung diseases.

In the present study, we sought to evaluate the effects of WT1 in fibroproliferation, myofibroblast differentiation, and ECM production in pulmonary fibrosis. We performed pulse-chase studies using lineagetracing mice for WT1-positive cells to investigate their fate in the pathogenesis of pulmonary fibrosis. Our findings show WT1 is a master regulator of multiple profibrotic processes, including proliferation, myofibroblast transformation, and ECM production. Notably, WT1-positive mesothelial cells lining the lungs transform into myofibroblasts. These data show for the first time to our knowledge that WT1 functions as a positive regulator of fibrosis in peripheral areas of the lung by influencing profibrotic functions of WT1negative fibroblasts in the pathogenesis of pulmonary fibrosis.

\section{Results}

The lineage tracing of postnatal WT1-positive mesothelial cells demonstrates mesothelial-to-myofibroblast transformation in vivo. WT1 is highly expressed in the lung during embryonic stages of lung development but is downregulated later in adult lungs (13). To determine the kinetics of WT1 expression during lung development, we collected lung tissues from E12.5 embryos through 3-week-old adult mice. WT1 was developmentally regulated in murine lungs with higher expression at E12.5 and E13.5, but it was significantly downregulated from E14.5 to postnatal stages, with the maintenance of low expression levels throughout adulthood (Figure 1A). To identify which cell types within embryonic lungs express a WT1 protein, immunostaining was performed on E15.5 lung sections from $\alpha$ SMA reporter mice with antibodies directed against WT1 and calretinin, a mesothelial cell marker. At E15.5, WT1 was selectively expressed by mesothelial cells lining the lung (calretininpositive) but it was not detected in myofibroblasts (Figure 1B). Indeed, the lung sections show colocalized expression of WT1 and calretinin in mesothelial cells lining the embryonic lungs at E15.5 (Figure 1C). WT1 protein is undetectable in pulmonary epithelial cells or endothelial cells in embryonic or adult lungs (data not shown). In contrast, WT1 was upregulated in mesothelial cells, fibroblasts, and myofibroblasts of subpleural fibrotic lesions in IPF and mouse models of TGF $\alpha$-induced pulmonary fibrosis $(22,25)$.

Recent studies from our group and others have shown that WT1 $1^{\text {CreERT2/- }}$ mice can be used to track the genetic lineage of WT1-expressing cells $(16,22)$. Upon WT1 expression, cells undergo Cre-driven genetic recombination to activate EGFP expression in WT1-expressing cells. The lineage-tracing studies suggest that WT1-positive mesothelial cells of embryonic lungs can give rise to mesenchymal cells that populate in the lung parenchyma. To demonstrate whether postnatal mesothelial cells of the lung transform to 
A

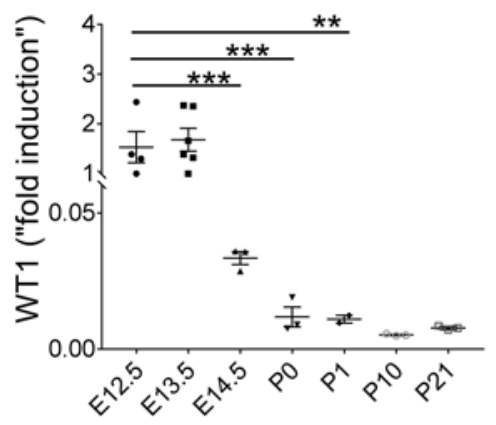

B

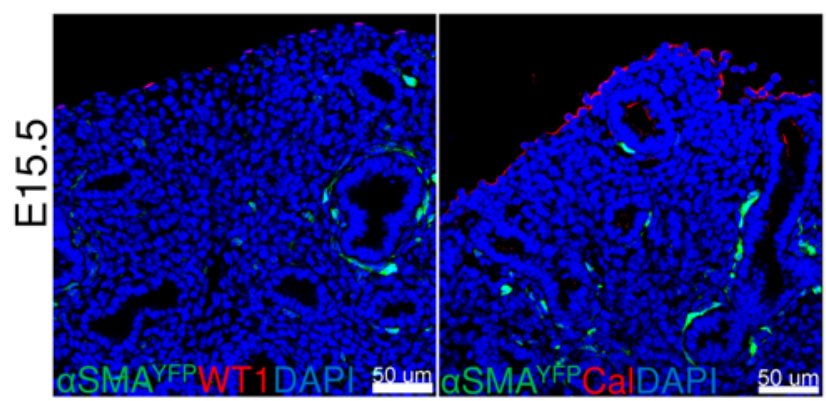

C

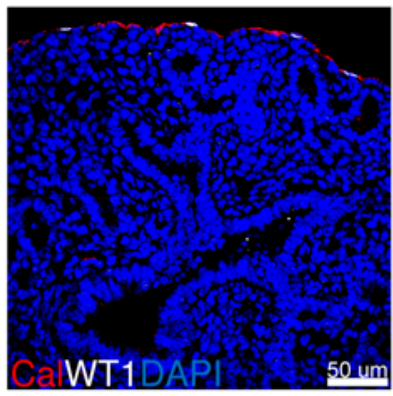

D

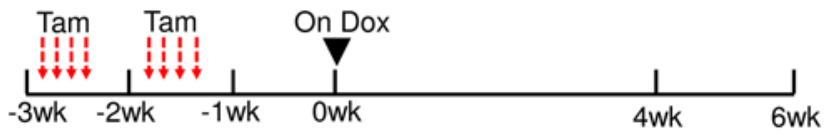

E

Pre-Tam

Control 4wk Dox

TGFa 4wk Dox

Pre-Tam
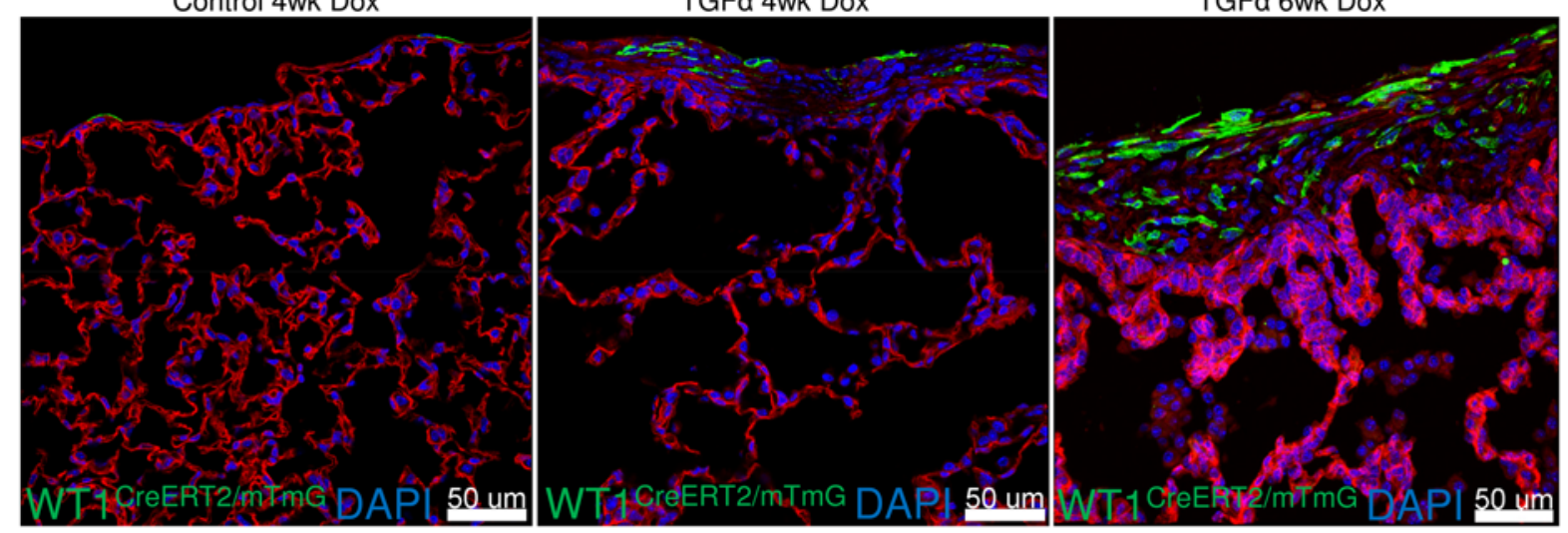

$\mathbf{F}$

Pre-Tam

TGFa 4wk Dox

Pre-Tam

TGFa 4wk Dox
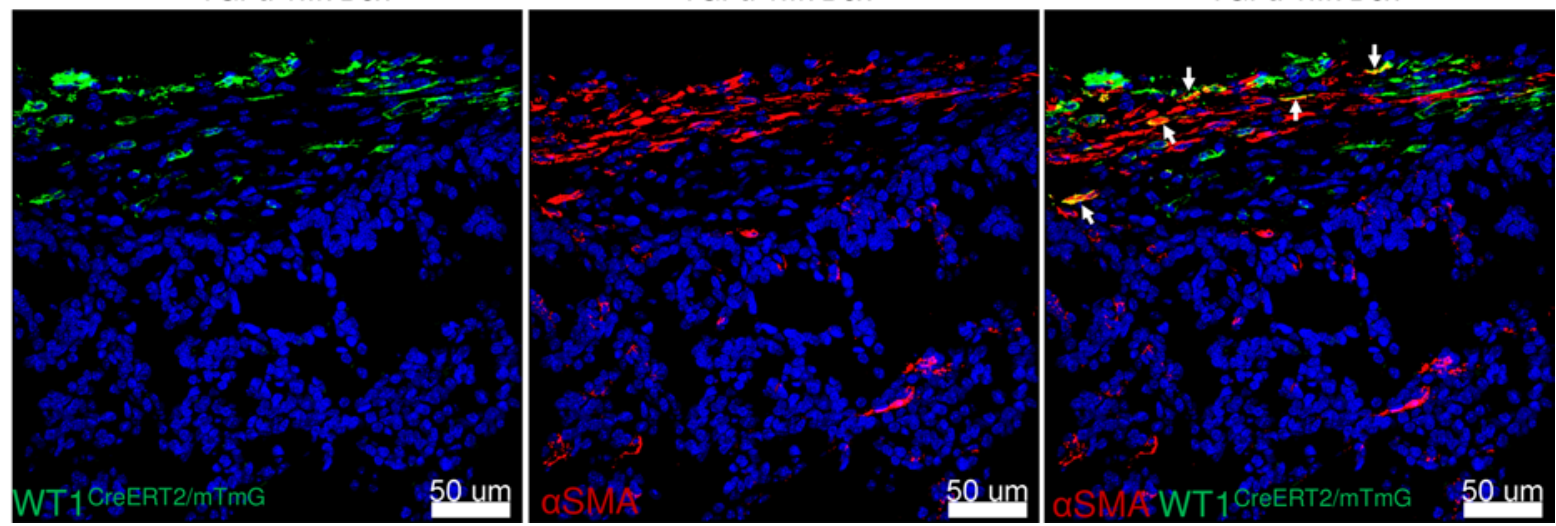

Figure 1. Postnatal WT1-positive mesothelial cell contributions to myofibroblasts in pulmonary fibrosis. (A) The dramatic decrease in the expression of WT1 in developing mouse lung at E14.5 and expression levels were normalized to HPRT mRNA ( $n=3-5 /$ gestational age). Data are presented as mean \pm SEM. Statistical significance was calculated using 1-way ANOVA with Sidak's multiple comparison for multiple comparisons. ${ }^{* *} P<0.005,{ }^{* * *} P<0.0005$. 
(B) Immunostaining shows the presence of WT1 protein in mesothelial cells (pleural surface) that coexpress calretinin (red) but not in myofibroblasts (green) of $\alpha S_{M A}{ }^{\mathrm{VFP}}$ mice embryos at E15.5. Scale bar: $50 \mu \mathrm{m}$. (C) WT1 staining (white) is detected in a subset of mesothelial cells positive for calretinin (red) in WT mice embryos at E15.5. Scale bar: $50 \mu \mathrm{m}$. (D) Schematic diagram of treatments with tamoxifen and Dox. Control or TGF $\alpha /$ WT1 ${ }^{\text {CreERT2/mTm }}$ mice were induced with tamoxifen, and 1 week later, pulmonary fibrosis was induced by administering Dox in food for 4 or 6 weeks. (E) Immunofluorescence images show progressive accumulation of WT1-derived cells residing in subpleura but not adventitia in TCF $\alpha /$ WT1 ${ }^{\text {CreERT2/mTmG }}$ mice compared with control WT1 ${ }^{\text {CreERT2/ }}$ ${ }_{\mathrm{mTmG}}$ mice on Dox for 4 or 6 weeks. Scale bar: $50 \mu \mathrm{m}$. (F) Immunostaining shows the presence of $\alpha$ SMA protein in WT1-positive mesothelium-derived mes-

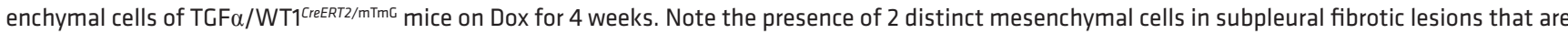
marked by $\alpha$ SMA protein in GFP-positive cells (WT1-positive mesothelial cells) and GFP-negative (WT1-positive mesenchymal cells) cells. Scale bar: 50 m.

myofibroblasts, we generated WT1 reporter mice (WT1 ${ }^{\text {CreERT2/mTmG }}$ mice) to trace the fate of WT1-positive mesothelial cells in adult mice during TGF $\alpha$-induced pulmonary fibrosis. To label WT1-positive mesothelial cells in healthy adult mice, tamoxifen was administered for 4 days per week for a total of 2 weeks, and a week later, mice were kept on doxycycline (Dox) food for 4 or 6 weeks to cause moderate to severe fibrotic lesions by TGF $\alpha$ overexpression in vivo (Figure 1D). Confocal image analysis of the subpleural fibrotic lesions shows a progressive increase in mesenchymal cells that originate from WT1-expressing postnatal mesothelium in subpleural fibrotic lesions (Figure 1E). However, green cells derived from WT1positive mesothelium were restricted to subpleural fibrotic lesions and did not populate in alveolar and adventitial areas of established fibrotic lesions with 6 weeks on Dox (Supplemental Figure 1, A and B; supplemental material available online with this article; https://doi.org/10.1172/jci.insight.121252DS1). To further establish mesothelium-to-myofibroblast transformation in vivo, the lung sections of WT1 ${ }^{\text {cre }}$ ERT2/- reporter mice on Dox for 6 weeks were immunostained with antibodies against $\alpha$ SMA. We detected several $\alpha$ SMA-positive green cells present in subpleural fibrotic lesions of TGF $\alpha / \mathrm{WT} 1{ }^{\text {CreERT2/mTmG }}$ mice on Dox for 6 weeks, indicating that some myofibroblasts were derived from mesothelial cells (Figure 1F). We observed many aSMA-negative green cells (likely fibroblasts) that may have originated from mesothelial cells. Also, we observed an accumulation of myofibroblasts that derived from WT1-negative mesothelial cells in subpleural fibrotic lesions of TGF $\alpha / \mathrm{WT} 1^{\text {CreERT2/mTmG }}$ mice on Dox for 6 weeks. The above findings suggest accumulation of mesothelial cell- and nonmesothelial cell-derived myofibroblasts in subpleural fibrotic lesions. These data are consistent with our previous data showing that WT1 is reactivated in both mesothelial cells and mesenchymal cells (fibroblast and myofibroblasts) in IPF and mouse models of TGF $\alpha$-induced pulmonary fibrosis.

WT1 lineage cells are the major source of mesenchymal cells in subpleural fibrotic lesions. To determine whether WT1 lineage cells contribute to the majority of mesenchymal cells, we treated TGF $\alpha / \mathrm{WT}^{\mathrm{CreERT} / \mathrm{mTmG}}$ mice or WT1 $1^{\text {CreERT2/mTmG }}$ control mice with tamoxifen for 4 days per week for a total of 2 weeks before Dox food (before tamoxifen; Pre-Tam) or continued with tamoxifen treatments at week 2 and 4 on Dox (Pre-Tam or after tamoxifen [Post-Tam]) (Figure 2A). The lungs were cultured for 7 days and were then analyzed by flow cytometry for GFP-positive fibroblasts (Supplemental Figure 2). In support of our hypothesis, we observed a significant increase in GFP-positive mesenchymal cells in lungs cultured from mice treated with tamoxifen continuously $(3.1 \% \pm 0.12 \%)$ compared with Pre-Tam-treated TGF $\alpha /$ WT $11^{\text {CreERT2 } / \mathrm{mTmG}}(1.75 \% \pm$ $0.05 \%)$ or $\mathrm{WT} 1^{\mathrm{CreERT} 2 / \mathrm{mTmG}}$ control mice $(0.3 \% \pm 0.00 \%)$ on Dox for 4 weeks (Figure $2, \mathrm{~B}$ and $\mathrm{C}$ ). In addition, confocal imaging of lung sections shows that the majority of mesenchymal cells that accumulate in subpleural fibrotic lesions were derived from WT1-positive postnatal lung cells (Figure 2D). Moreover, we observed a significant increase in WT1-positive cells in reporter mice that were treated with tamoxifen

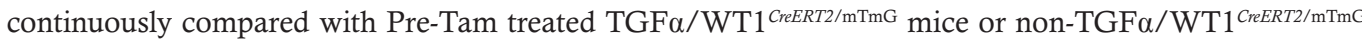
mice on Dox for 4 weeks. Taken together, our results demonstrate that most mesenchymal cells within subpleural fibrotic lung lesions originated from WT1-positive mesothelial cells and fibroblasts. Both the above populations were localized in subpleural fibrotic lesions but not in lung parenchyma or adventitia (Supplemental Figure 1B). Together, these findings suggest that myofibroblasts that accumulate in subpleural fibrotic lesions following TGF $\alpha$ overexpression are derived from both mesothelial cells and lung-resident fibroblasts, perhaps through increased induction of WT1 in subpleural fibrotic lesions. These findings further support fibroblast heterogeneity and possible cross-talk between multiple mesenchymal cell types in the pathogenesis of the severe fibrotic disease.

WT1 is a positive regulator of fibroblast-to-myofibroblast transformation. Progressive accumulation of myofibroblasts is an essential determinant of pulmonary fibrosis, as they are responsible for pathological contractures and the excessive ECM deposition in the mature fibrotic lesions of human IPF (26-29). We observed a significant 
A
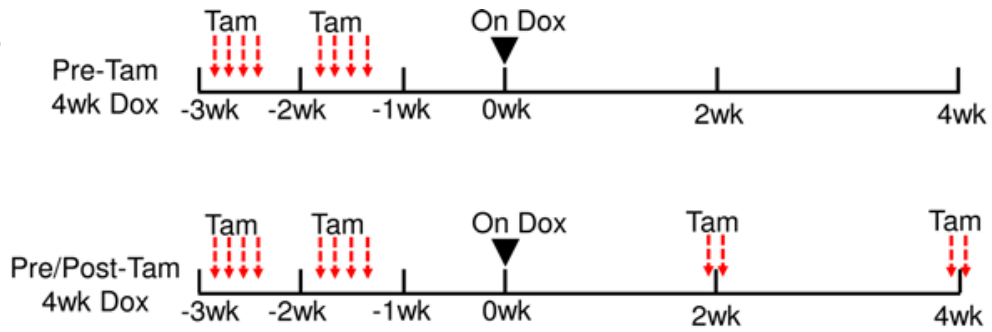

B Pre-Tam
ontrol 4wk Dox

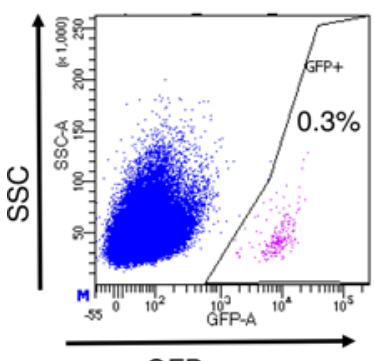

GFP
D

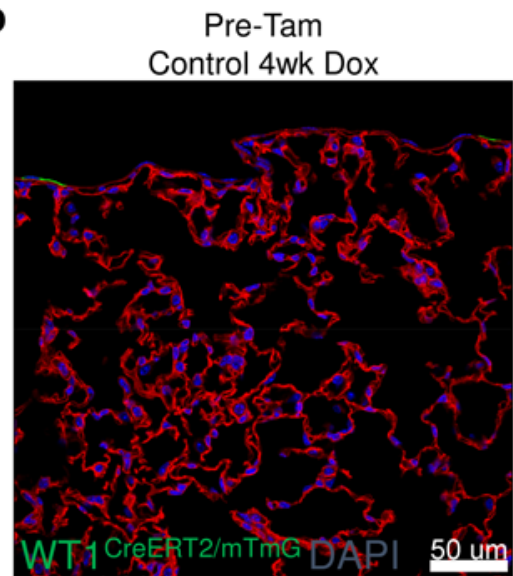

Pre-Tam TGFa 4wk Dox

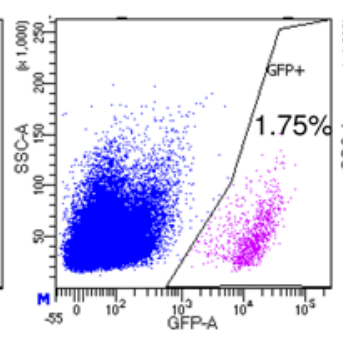

Pre/Post-Tam TGFa 4wk Dox

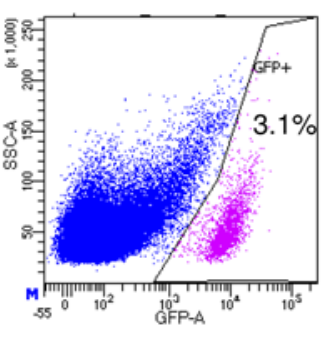

C

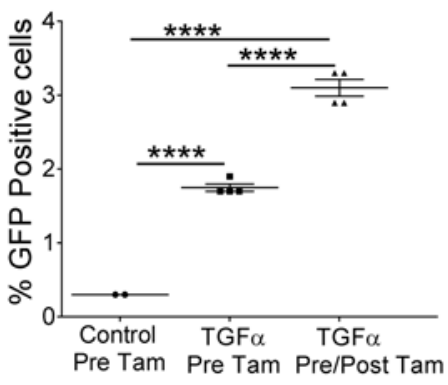

Figure 2. WT1-lineage cells are the major source of mesenchymal cells in subpleural fibrotic lesions in vivo. (A) Schematic diagram of treatments with

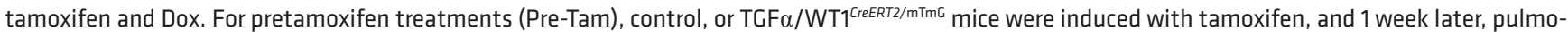
nary fibrosis was induced by administering Dox in food for 4 weeks. For Pre- and posttamoxifen treatments (Pre/Post-Tam), TGF $\alpha /$ WT1 ${ }^{\text {CreERT2/mTm }}$ mice were treated with tamoxifen before and after pulmonary fibrosis was induced by Dox in food for 4 weeks. (B) Representative FACS plots show increased accumulation of WT1-drived myofibroblasts in Pre-/Post-Tam-treated TCF $\alpha /$ WT1 1 reERT2/mTmG mice compared with Pre-Tam-treated TCF $\alpha /$ WT1 1 reERT2/mTmG mice or control mice on Dox for 4 weeks. (C) Quantification of GFP-positive myofibroblasts in lung cultures of tamoxifen-treated TCF $\alpha /$ WT ${ }^{\text {creeRT2/mTmG }}$ mice or control mice on Dox for 4 weeks. (D) Immunofluorescence images show increased accumulation of WT1-derived mesenchymal cells residing in subpleura

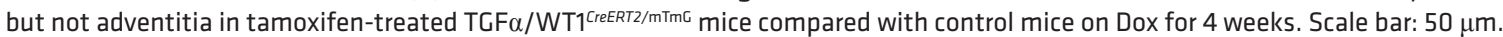

increase in the transcript levels of WT1 and $\alpha \mathrm{SMA}$ in primary fibroblasts isolated from fibroblast cultures of non-IPF lungs infected with WT1-overexpressing virus compared with control virus infections (Figure 3A). Also, we detected a significant increase in $\alpha$ SMA protein levels in cells infected with WT1-overexpressing virus compared with control virus infections (Figure 3B). Additionally, lentivirus-mediated (lenti-mediated) overexpression of WT1 was sufficient to increase the aSMA expression in murine lung-resident fibroblasts (Figure 3C).

To evaluate the role of WT1 in fibroblast-to-myofibroblast transformation, we used a cell fate-mapping strategy based on lineage-specific expression of $\alpha \mathrm{SMA}$ in lung-resident fibroblasts isolated from $\alpha \mathrm{SMA}$ reporter mice ( $\alpha \mathrm{SMA}^{\mathrm{CrERT2} / \mathrm{mTmG}}$ mice). In this model, activation of Cre recombinase under control of $\alpha \mathrm{SMA}$ promoter removes the STOP cassette between the 2 loxP sites, which irreversibly changes the expression of membrane tomato $(\mathrm{mT})$ to membrane GFP (mG) in aSMA-expressing lung-resident fibroblasts. We isolated the lung-resident fibroblasts from lung cultures of $\alpha$ SMA reporter mice and transduced each with either 
A

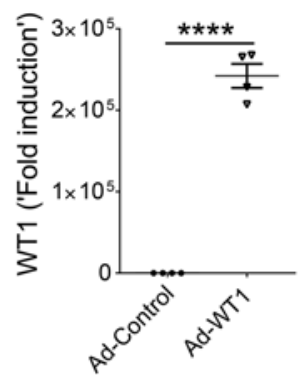

C

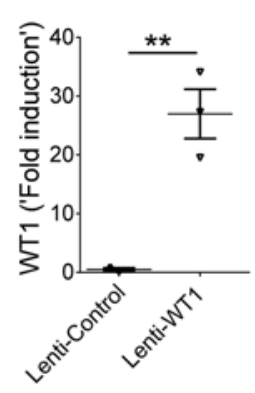

E

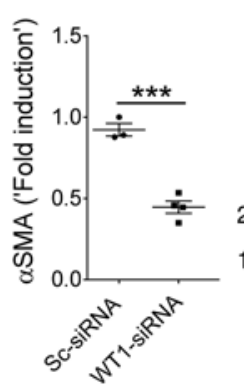

$\mathbf{F}$

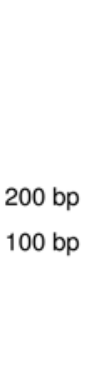

B
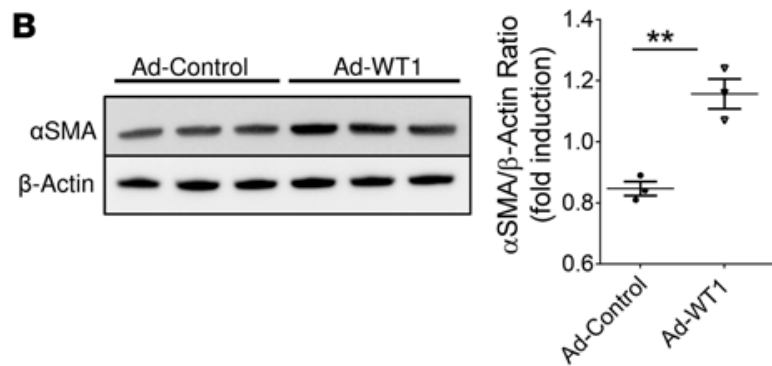

D
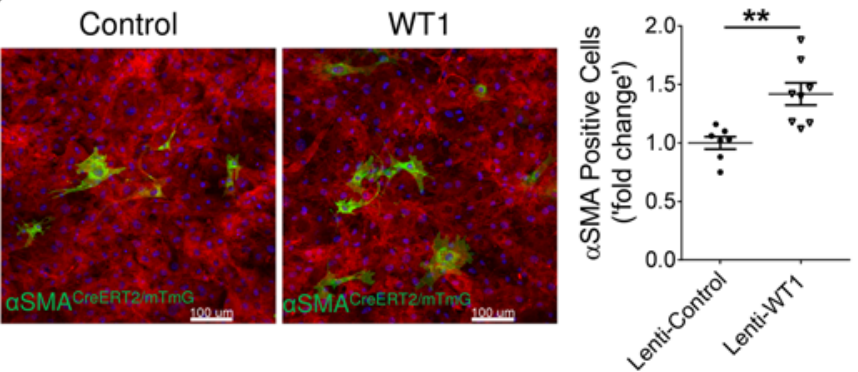

G

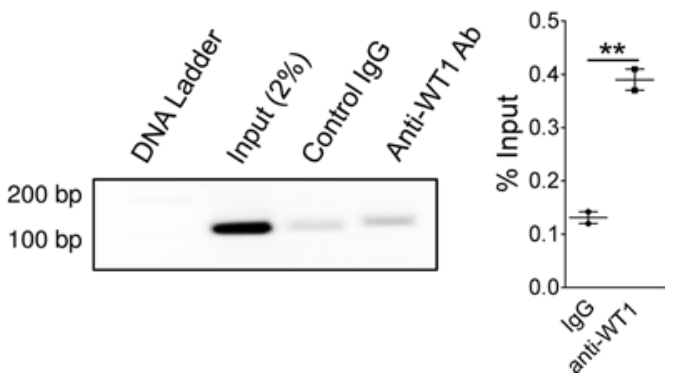

Figure 3. WT1 is a positive regulator of fibroblast-to-myofibroblast transformation. (A) Human non-IPF fibroblasts were transduced with either control adenovirusor WT1 adenovirus for 24 hours. Transcripts of WT1 and $\alpha$ SMA were quantified using RT-PCR $(n=6)$. Results are cumulative, from 2 independent experiments with similar results. (B) Human non-IPF fibroblasts were transduced with either control adenovirusor WT1 adenovirus for 72 hours. Protein lysates were immunoblotted for $\alpha$ SMA and $\beta$-actin. $\alpha$ SMA quantification was performed by normalizing to the endogenous $\beta$-actin control. Results are representative of 2 independent experiments with similar results $(n=3)$. (C) Fibroblasts of nontransgenic mice on Dox for 4 weeks were transduced with either control lentivirus or WT1 lentivirus (10 MOI) for 24 hours. Transcripts of WT1 and $\alpha$ SMA were quantified using RT-PCR ( $n=$ 3). Results are representative of 3 independent experiments with similar results. (D) Fibroblasts of $\alpha S M A^{\text {CreERTz/mTmG }}$ mice were transduced with either control lentivirus or WT1 lentivirus for 72 hours in the presence of 4-hydroxy tamoxifen. Immunofluorescence images were obtained at an original magnification of $\times 20$. Scale bar: $100 \mu \mathrm{m}$. The number of $\alpha$ SMA-positive (GFP-positive) myofibroblasts were quantified in images, and the data shown are cumulative of 2 independent experiments with similar results $(n=4)$. (E) Fibroblasts from IPF primary lung cultures were transiently transfected with either control or WT1-specific siRNA for 72 hours, and $\alpha$ SMA gene expression was analyzed by RT-PCR. Results are representative of 2 independent experiments with similar results $(n=4)$. (F) IMR-90 cells were transduced with WT1 adenovirus (100 MOI) for 72 hours. Cell lysates were prepared, and the ChIP assay was performed with anti-WT1 antibody or normal rabbit IgG as a negative control using $\alpha$ SMA gene promoter-specific PCR primers. Nonimmunoprecipitated DNA is represented as input DNA (product size, $140 \mathrm{bp)}$ ). (G) Primary lung-resident fibroblasts were isolated from lung cultures of TCF $\alpha$ mice placed on Dox for 8 weeks. Cell lysates were prepared, and the ChIP assay was performed with anti-WT1 antibody or normal rabbit IgG as a negative control using $\alpha$ SMA gene promoter-specific PCR primers. Nonimmunoprecipitated DNA is represented as input DNA (product size, 104 bp). Data are representative of 2 independent experiments. Data are presented as mean \pm SEM. Unpaired Student $t$ test, ${ }^{*} P<0.05$, ${ }^{* *} P<0.005$, ${ }^{* * *} P<$ $0.0005,{ }^{* * * *} P<0.0001$.

control or WT1-overexpressing lentivirus in the presence of hydroxy-tamoxifen. We observed a significant increase in the number of transformed green cells, showing a fibroblast-to-myofibroblast transformation (Figure 3D). Further, the knockdown of WT1 significantly diminished the $\alpha$ SMA gene expression in lungresident fibroblasts isolated from fibrotic lungs of IPF patients (Figure 3E).

To determine whether the observed increases in $\alpha$ SMA levels and myofibroblast transformation could be due to direct or indirect effects by WT1, we performed a computational analysis of the sequence of human and mouse $\alpha$ SMA promoter regions for possible WT1 binding sites, which revealed the presence of 
WT1 binding sites that are highly conserved among mammals, including human and mouse $\alpha$ SMA genes (Supplemental Figure 3). ChIP analysis in WT1-adenoviral-transfected IMR-90 fibroblasts using WT1 specific antibodies revealed that the WT1 bound more efficiently to the $\alpha$ SMA gene (Figure $3 F$ ), and no signal was observed with control isotype antibody, suggesting that this site may play an important role in WT1mediated $\alpha$ SMA expression. Similarly, we performed ChIP analysis of $\alpha$ SMA promoter occupancy by WT1 in primary lung-resident fibroblasts isolated from TGF $\alpha$ mice placed on Dox for 8 weeks. ChIP analysis revealed that WT1 bound more efficiently to the $\alpha$ SMA gene compared with control isotype antibody (Figure $3 \mathrm{G}$ ). Together, these data confirm that $\alpha \mathrm{SMA}$ gene expression during myofibroblast transformation was mediated by binding of the WT1 transcription factor to the aSMA gene promoter. Taken together, our in vitro results establish that WT1 upregulation in lung-resident fibroblasts augments the transformation of fibroblasts to myofibroblasts.

WT1 is a positive regulator of profibrotic gene transcription and fibroproliferation in IPF. The loss of WT1 transcripts has resulted in reduced expression of multiple ECM genes in fibroblasts isolated from IPF lungs or mice with severe fibrotic lung disease (22). To gain insight into the global change in gene transcription due to WT1, we isolated fibroblasts from the lungs of transgenic mice carrying a Dox-regulated transgene leading to TGF $\alpha$ expression in lung epithelium. RNA sequencing (RNA-sequencing; RNA-Seq) showed that the knockdown of WT1 altered the expression of 2,425 genes, half of which were induced (1,193 genes) and half of which were repressed (1,232 genes) by WT1 (Supplemental Figure 4). To identify profibrotic gene transcripts altered by WT1 in IPF, we performed an enrichment analysis of the negatively correlated gene sets between IPF lungs and WT1-specific siRNA-treated lung fibroblasts (30). Gene expression profiles of IPF lungs from a previously published transcriptomic data set (8) (Gene Expression Omnibus [GEO] accession number GSE53845; https://www.ncbi.nlm.nih.gov/geo/query/acc.cgi) were compared with that of WT1 altered genes in fibroblasts, and we identified multiple transcripts that were either upregulated (107 genes) or downregulated (63 genes) by WT1 in IPF (Supplemental Figure 5A, Supplemental Table 1). Using ToppFun application of the ToppGene Suite (31), the top enriched biological processes common to both the IPF and WT1-specific data sets were proliferation, mesenchyme growth, development, and ECM production in IPF (Supplemental Figure 5B). To validate WT1-driven genes in fibroblasts, we performed reverse transcription PCR analysis of several ECM genes. Aside from WT1, we noted a significant reduction in transcripts for Col14, Col15, Itg2, Itg7, and Lum, suggesting that the maintenance of ECM is WT1 dependent (Supplemental Figure 5C).

Lung-resident fibroblasts have been shown to proliferate and serve as one of the major mesenchymal cell sources in the fibrotic lung lesions of IPF and mouse models of pulmonary fibrosis (32-37). Likewise, we observed higher proliferation capacity of lung-resident fibroblasts isolated from IPF lungs compared with fibroblasts isolated from normal lungs (Supplemental Figure 6). To determine the role of WT1 in fibroproliferation, we overexpressed WT1 in primary lung-resident fibroblasts isolated from normal lungs and quantified changes in proliferation. In order to clarify the status of WT1 in adenovirus-transduced cells, we performed Western blot analysis to demonstrate high amounts of WT1 in WT1-adenovirus-transduced cells compared with empty-adenovirus-transduced cells (Figure 4A). Adeno-mediated overexpression of WT1 caused a significant increase in the amount of proliferative cell nuclear antigen (PCNA) in lung-resident fibroblasts compared with cells infected with empty adenovirus (Figure 4A). Also, the knockdown of WT1 was sufficient to attenuate proliferation of lung-resident fibroblasts isolated from IPF lungs (Figure 4B). Lenti-mediated overexpression of WT1 increased the proliferative capacity of murine lung-resident fibroblasts (Figure 4C). Furthermore, the knockdown of WT1 significantly diminished proliferation of lung-resident fibroblasts isolated from fibrotic lungs of TGF $\alpha$ transgenic mice on Dox for 4 weeks (Figure 4D). Further, we observed a significant decrease in the number of PCNA-positive mouse fibroblasts with knockdown of WT1 using WT1-specific siRNA compared with control siRNA (Supplemental Figure 7). Together, these data demonstrate that the proliferative capacity of lung-resident fibroblasts was largely dependent on WT1 expression and indicates a positive regulation of fibroproliferation by WT1. Levels of WT1-driven proliferative genes in IPF, including Grem1, Runx1, Wnt4, Stat3, Prrx1, Igf1, Ccnb1, and E2f8 were reduced in primary lung-resident fibroblasts of TGF $\alpha$ mice on Dox for 4 weeks treated with WT1 siRNA compared with those treated with control siRNA (Figure 4E). Notably, the genetic knockdown of WT1 was sufficient to attenuate the expression of WT1-driven proliferative genes that are associated with IPF. These findings establish that WT1 functions as a positive regulator of fibroproliferation in IPF. 
A

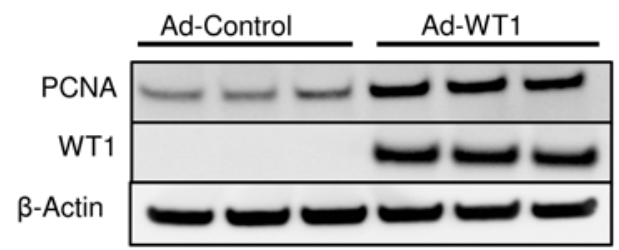

C

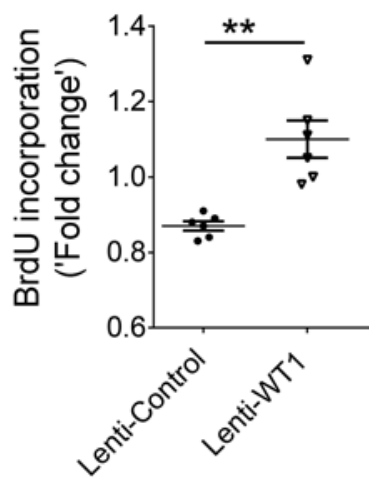

D

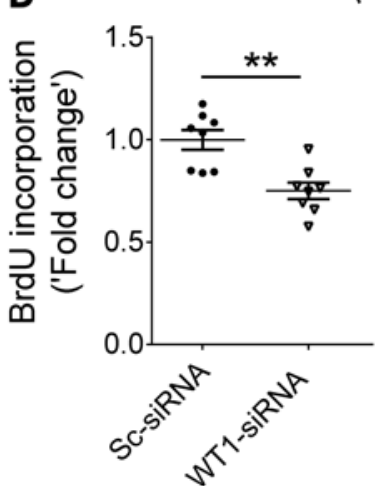

B

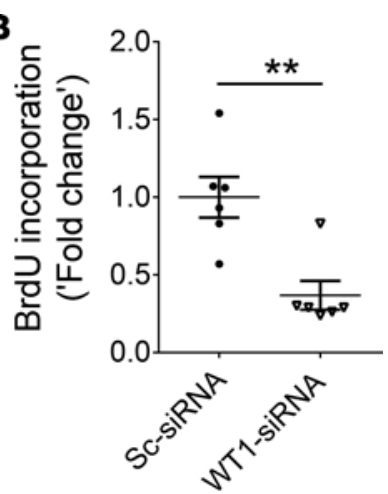

E

Grem1

Runx1
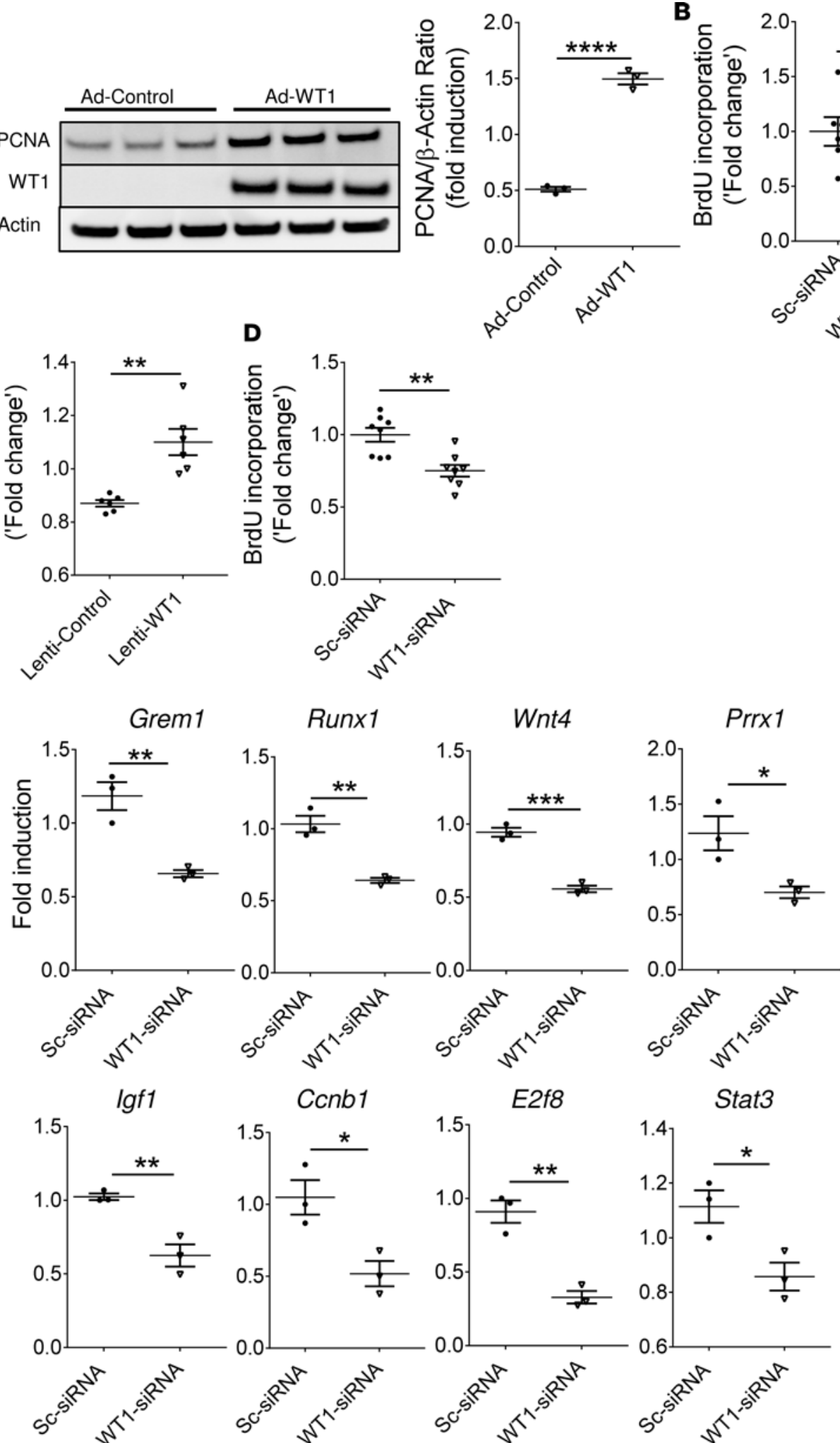

Ccnb1

E2f8

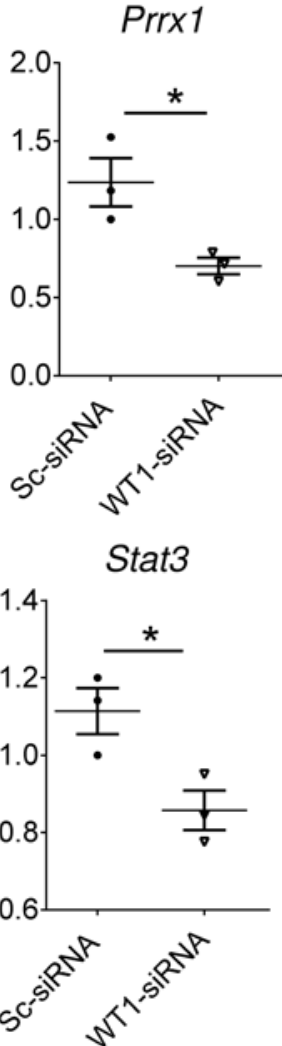

Prrx1
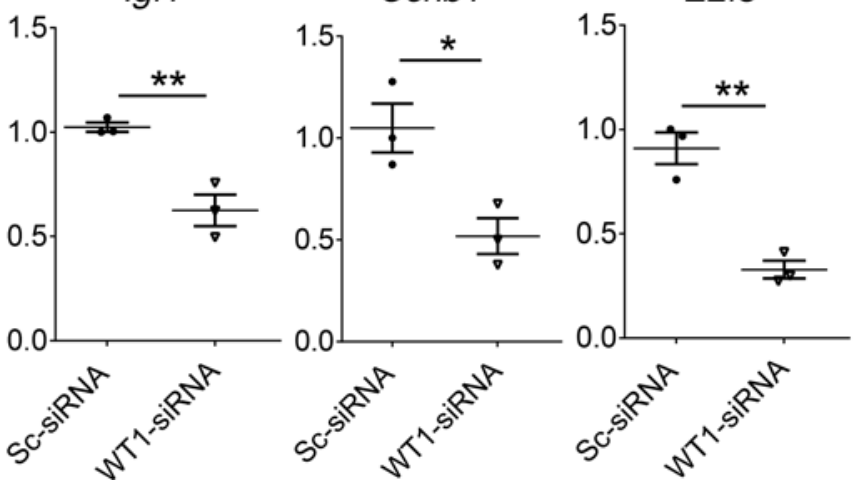

Figure 4. WT1 is a positive regulator of fibroproliferation. Primary lung-resident fibroblasts were isolated from the lung cultures of IPF, non-IPF, WT, or TCF $\alpha$ transgenic mice by negative selection using ant-CD45 magnetic beads. (A) Human non-IPF fibroblasts were transduced with either control adeno or WT1 adenovirus for 72 hours. Proliferation was assessed by immunoblotting WT1 and proliferating cell nuclear antigen (PCNA). PCNA quantification was performed by normalizing to the endogenous $\beta$-actin control. Results are representative of 2 independent experiments with similar results $(n=3)$. (B) Human IPF fibroblasts were transfected with either control or WT1 siRNA for 72 hours, and proliferation was assessed by BrdU incorporation. Results are cumulative of 2 independent experiments with similar results $(n=4)$. (C) Fibroblasts of nontransgenic mice on Dox for 4 weeks were transduced with either control lenti or WT1 lentivirus for 72 hours. Proliferation was assessed by BrdU incorporation assay. Results are representative of 2 independent 
experiments with similar results $(n=6)$. (D) Fibroblasts of TCF $\alpha$ transgenic mice on Dox for 4 weeks were transiently transfected with either control or WT1 siRNA for 72 hours, and proliferation was assessed by BrdU incorporation. Results are cumulative of 2 independent experiments with similar results ( $n=$ 4). (E) WT1-driven proliferative genes including Grem1, Runx1, Wnt4, Stat3, Prrx1, Igf1, Ccnb1, and E2f8 were quantified in fibroblasts of TCF $\alpha$ transgenic mice on Dox for 4 weeks transfected with either control or WT1 siRNA for 48 hours. Results are representative of 3 independent experiments with similar results $(n=3)$. Data are presented as mean \pm SEM. Unpaired Student $t$ test ${ }^{*} P<0.05,{ }^{* *} P<0.005,{ }^{* * *} P<0.0005,{ }^{* * * *} P<0.0001$.

The partial loss of WT1 was sufficient to attenuate pulmonary fibrosis in vivo. Our recent study demonstrated that conditional overexpression of TGF $\alpha$ or bleomycin-induced lung injury caused in WT1 upregulation and severe fibrotic lung disease with histologic features similar to human IPF $(22,38)$. Heterozygous WT1 $1^{\text {CreERT2/- }}$ mice (knock-in allele) are haploinsufficient for the WT1 allele (WT1 ${ }^{+/}$) but display uninterrupted normal lung development in embryonic and adult stages, with viability similar to WT mice. Loss of a WT1 allele had no effect on lung morphology or collagen deposition (Supplemental Figure 8). We crossed TGF $\alpha$ mice with $\mathrm{WT}^{+/-}$mice to generate a unique set of double- and triple-transgenic mice carrying mutant $\mathrm{WT} 1\left(\mathrm{WT}_{1}^{+/-}\right.$and $\left.\mathrm{TGF} \alpha / \mathrm{WT}^{+/-}\right)$or WT $\mathrm{WT} 1\left(\mathrm{WT} 1^{+/+}\right.$and $\left.\mathrm{TGF} \alpha / \mathrm{WT} 1^{+/+}\right)$. To identify whether WT1 deficiency influences fibrosis susceptibility, mice were placed on Dox for 4 weeks, a time period that leads to significant lung fibrosis upon overexpression of TGF $\alpha$. The loss of 1 allele of WT1 was sufficient to attenuate subpleural fibrosis in Dox-treated TGF $\alpha / \mathrm{WT}^{+/-}$mice compared with $\mathrm{TGF} \alpha /$ $\mathrm{WT}^{+/+}$mice (Figure 5A). To determine if WT1 haploinsufficiency affects rtTA-driven TGF $\alpha$ transgene expression, we measured transcripts of TGF $\alpha$ in all 4 groups. As expected, we observed an increase in $\mathrm{TGF} \alpha$ transcripts in Dox-treated $\mathrm{TGF} \alpha / \mathrm{WT}^{+/+}$and $\mathrm{TGF} \alpha / \mathrm{WT} 1^{+/-}$mice, and $\mathrm{TGF} \alpha$ transcripts did not differ between mutant and WT WT1 mice (Figure 5B). In support of our hypothesis, WT1 transcripts were attenuated in the lungs of Dox-treated $\mathrm{TGF} \alpha / \mathrm{WT} 1^{+/-}$mice compared with $\mathrm{TGF} \alpha / \mathrm{WT} 1^{+/+}$mice (Figure 5C), and reduction of WT1 transcripts was associated with a significant decrease in lung hydroxyproline levels (Figure 5D), as well as improvement in lung compliance (Figure 5E). To further substantiate that WT1 haploinsufficiency contributes to diminished fibrotic responses during bleomycin-induced fibrosis, WT and WT1 haploinsufficient mice were treated by intradermal injections of bleomycin for 5 days a week for a total of 4 weeks (22). Attenuation of Masson's trichrome staining in both subpleural and parenchymal areas of lung sections was detected in $\mathrm{WT} 1^{+/-}$mice compared with $\mathrm{WT} 1^{+/+}$mice treated with bleomycin (Figure 5F). Also, the transcripts of $W t 1$, Coll $\alpha$, and Col5 $\alpha$ genes were reduced in bleomycin-treated $\mathrm{WT}^{+/-}$mice compared with $\mathrm{WT} 1^{+/+}$mice (Figure $5, \mathrm{G}-\mathrm{I}$ ), and lung function was improved in $\mathrm{WT}^{+/-}$mice compared with $\mathrm{WT} 1^{+/+}$mice (Figure $5 \mathrm{~J}$ ). Thus, our findings provide complementary and direct in vivo evidence that WT1 plays a pathogenic role in pulmonary fibrosis.

WT1 augments fibroproliferation and myofibroblast transformation in a non-cell autonomous manner. Using Ki67 as a marker of proliferation, we observed a notable decrease in Ki67-positive cells in both subpleural and adventitial areas in the lungs of $\mathrm{TGF} \alpha / \mathrm{WT} 1^{+/-}$compared with $\mathrm{TGF} \alpha / \mathrm{WT} 1^{+/+}$mice on Dox for 4 weeks (Figure 6A). Although WT1-expressing cells were localized to subpleural areas, we observed a significant decrease in the proliferation of parenchymal fibrotic lesions during TGF $\alpha$ - and bleomycin-induced pulmonary fibrosis. To identify myofibroblast accumulation, lung sections were immunostained with antibodies against $\alpha \mathrm{SMA}$. $\alpha \mathrm{SMA}$ staining is attenuated in both subpleural and adventitial areas in the lungs of Dox-treated TGF $\alpha / \mathrm{WT}^{+/+}$mice compared with $\mathrm{TGF} \alpha / \mathrm{WT}^{+/-}$mice (Figure $6 \mathrm{~B}$ ). Consistent with the above findings, we observed attenuation of myofibroblast accumulation in both subpleural and parenchymal areas of the lungs in $\mathrm{WT}^{+/+}$mice compared with $\mathrm{WT}^{+/-}$mice treated with bleomycin (Figure 6C). However, our recent studies demonstrated that WT1 expression is limited to subpleural areas of fibrotic lungs during TGF $\alpha$ - and bleomycin-induced pulmonary fibrosis (22).

To investigate possible crosstalk between WT1-positive and WT1-negative fibroblasts, we generated a potentially novel multitransgenic WT1 reporter mice by serial breeding of TGF $\alpha$ transgenic mice with

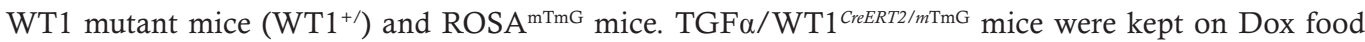
for 4 weeks, and lung-resident fibroblasts were isolated and treated with either control or WT1-specific siRNA and cultured with tamoxifen to identify the proliferative changes in both WT1-positive and WT1negative lung-resident fibroblasts (Figure 6D). As expected, we observed a significant decrease in the number of PCNA-positive mouse fibroblasts and a decrease in WT1-expressing cells with the knockdown of WT1 compared with control siRNA (Figure 6, D and E). Notably, the proliferation was reduced in both $\mathrm{WT}^{+} / \mathrm{PCNA}^{+}$and $\mathrm{WT}^{-} / \mathrm{PCNA}^{+}$cells (Figure $6, \mathrm{~F}$ and $\mathrm{G}$ ). Further, we performed transwell coculture studies in which WT1-overexpressing lung-resident fibroblasts isolated from TGF $\alpha$ mice on 
A $\mathrm{TGFa} / \mathrm{WT} 1^{+/+}$
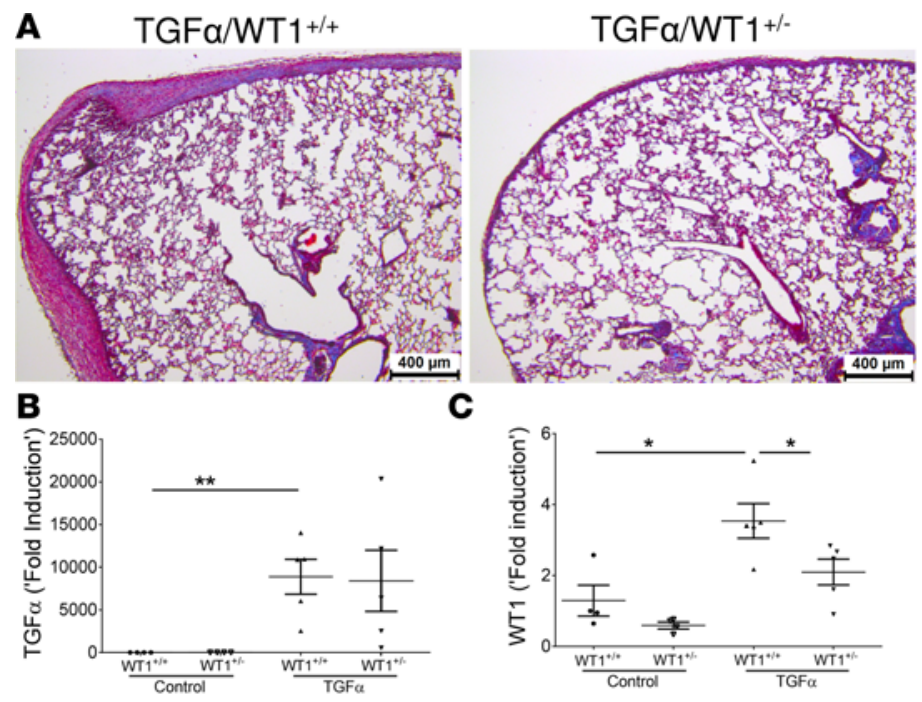

C

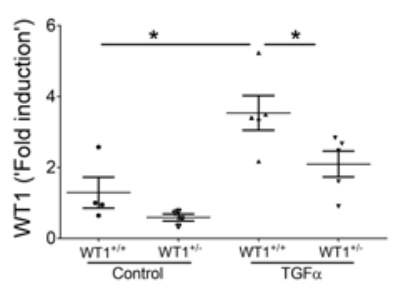

D
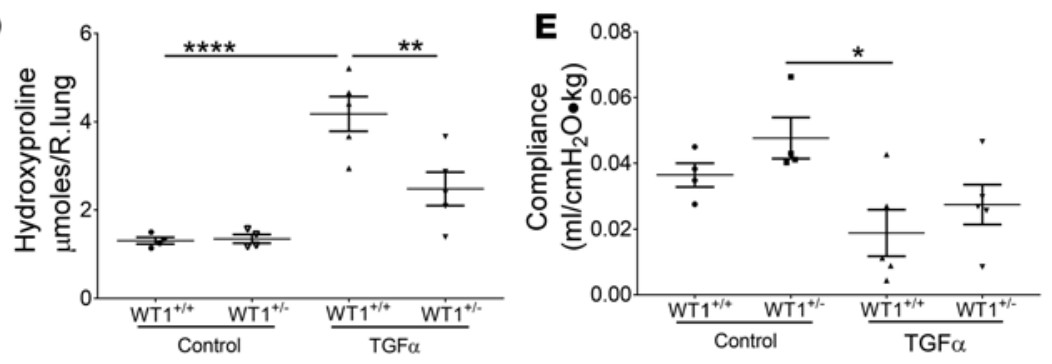

$\mathbf{F}$

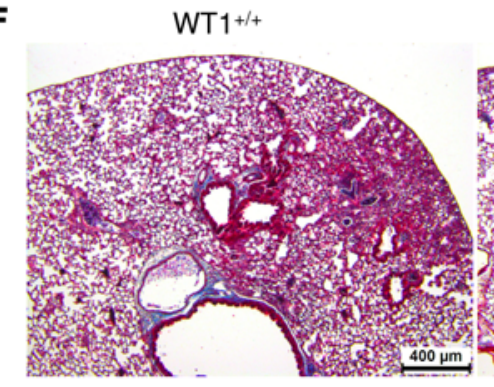

G

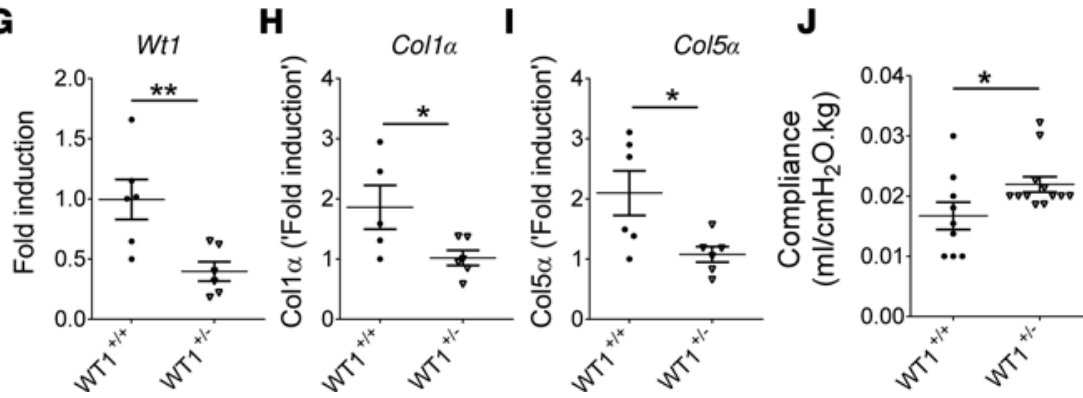

Figure 5. The partial loss of WT1 attenuates pulmonary fibrosis in vivo. CCSP/rtTA and TetO/TCF $\alpha$ transgenes were bred into WT1 ${ }^{\text {reERT2/- }}$ knock-in mice $\left(\mathrm{WT}^{+/-}\right)$to generate TGF $\alpha$ transgenic mice with WT or mutant WT1 allele, and all groups of mice were administered Dox for 4 weeks. In bleomycin model, $\mathrm{WT}^{+/+}$and $\mathrm{WT}^{+/-}$mice were treated with bleomycin ( 6 units per kg body weight) in $50 \mu \mathrm{l}$ of saline solution intradermally (i.d.) for 5 days per week for a total of 4 weeks. (A) Masson's trichrome-stained lung sections of TCF $\alpha / \mathrm{WT}^{+/+}$and TCF $\alpha / \mathrm{WT}^{+/-}$ mice on Dox for 4 weeks ( $n=4-5 /$ group). Image magnification, $\times 5$. Scale bar: $400 \mu \mathrm{m}$. (B) TCF $\alpha$ transcripts were measured in WT (WT1 $1^{++}$and TGF $\alpha$ ) $\left.\mathrm{WT}^{+/+}\right)$and WT1 mutant (WT1 ${ }^{+/-}$and TGF $\alpha / \mathrm{WT}^{+/}$) mice on Dox for 4 weeks ( $n=4-5 /$ group). (C) WT1 transcripts were measured in WT (WT1 $1^{++}$and TGF $\alpha$ ) $\left.\mathrm{WT}^{+/+}\right)$and WT1 mutant (WT1 ${ }^{+/-}$and $\left.\mathrm{TCF} \alpha / \mathrm{WT}^{+/-}\right)$ mice on Dox for 4 weeks ( $n=4-5 /$ group). (D) The total lung hydroxyproline levels in $\mathrm{WT}\left(\mathrm{WT} 1^{+/+}\right.$and $\left.\mathrm{TCF} \alpha / \mathrm{WT}^{+/+}\right)$and WT1 mutant (WT1 ${ }^{+/-}$and TCF $\alpha /$ $\mathrm{WT}^{+/-}$) mice on Dox for 4 weeks ( $n=4-5 /$ group). (E) The lung compliance was measured using FlexiVent in $\mathrm{WT}\left(\mathrm{WT}^{+/+}\right.$and $\left.\mathrm{TGF} \alpha / \mathrm{WT}^{+/+}\right)$and WT1 mutant (WT1 ${ }^{+/-}$and TCF $\alpha / \mathrm{WT}^{+/-}$) mice on Dox for 4 weeks ( $n=4-5$ /group). (F) Masson's trichrome-stained lung sections of $\mathrm{WT}^{+/+}$and $\mathrm{WT} 1^{+/-}$mice treated with bleomycin for 4 weeks ( $n=6 /$ group). Image magnification, $\times 5$. Scale bar: $400 \mu \mathrm{m}$. (C) WT1 transcripts were measured in the total RNA isolated from the lungs of $\mathrm{WT}^{+/+}$and $\mathrm{WT}^{+/-}$mice treated with bleomycin for 4 weeks ( $n=6 /$ group). (H and $\mathbf{I}$ ) Col1 $\alpha$ and Col5 $\alpha$ transcripts were measured in the total RNA isolated from the lungs of $\mathrm{WT}^{+/+}$and $\mathrm{WT}^{+/-}$mice treated with bleomycin for 4 weeks ( $n$ =6/group). (J) The lung compliance was measured using FlexiVent in $\mathrm{WT}^{+/+}$and $\mathrm{WT}^{+/-}$mice treated with bleomycin for 4 weeks ( $n=6$ /group). Results are representative of 2 independent experiments with similar results. Data are presented as mean \pm SEM. Statistical significance was calculated using 1-way ANOVA with Sidak's multiple comparison test or unpaired Student $t$ test for comparison between 2 groups. ${ }^{*} P<0.05,{ }^{* *} P<0.005,{ }^{* * *} P<0.0005$.

Dox for 6 weeks were cultured in an upper chamber and control lung-resident fibroblasts were placed in the bottom chamber (Figure 6H). Using immunostaining for PCNA and quantifying the PCNA-positive cells in the bottom chamber, the TGF $\alpha$ fibroblast increased PCNA-positive fibroblasts after 3 days of cocultures (Figure $6 \mathrm{H}$ ). To demonstrate that the above effect is specific to WT1 upregulation, we prepared conditioned media from primary fibroblasts isolated and treated with either control or WT1specific siRNA from TGF $\alpha$ mice on Dox for 6 weeks. We observed a significant decrease in the number of PCNA-positive fibroblasts with conditioned medium prepared from the TGF $\alpha$ fibroblasts treated with WT1-specific siRNA compared with control siRNA (Figures 6I). These findings show that WT1 can 
A

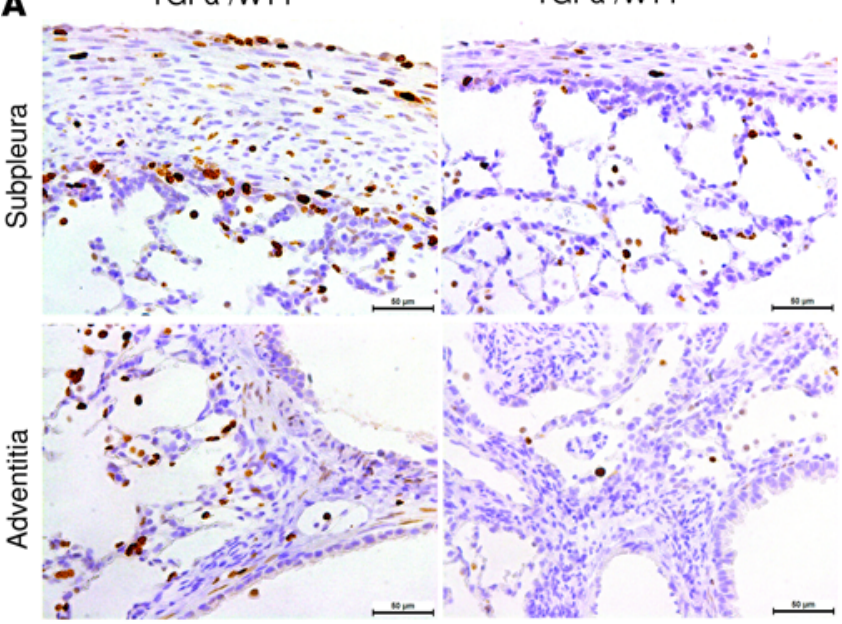

C

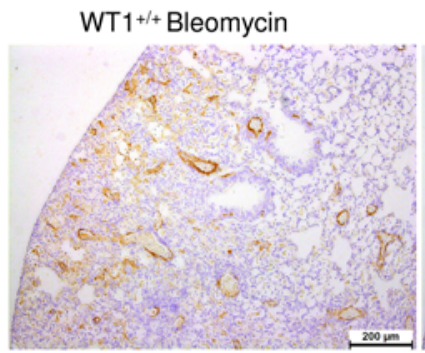

TGFa-/WT1+/-

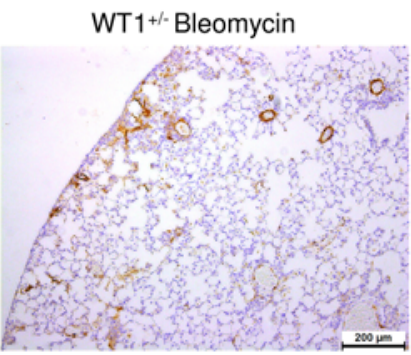

B

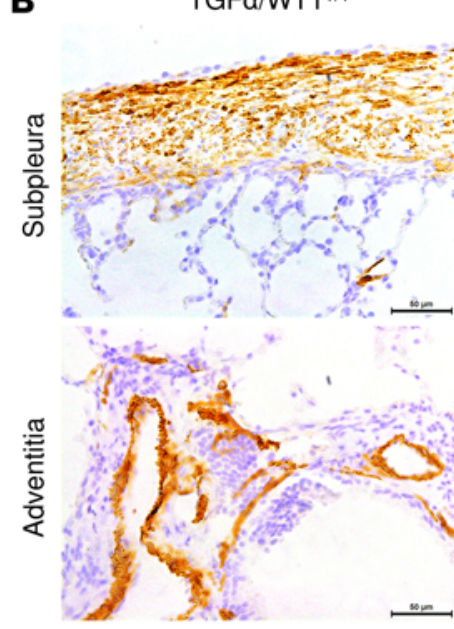

D

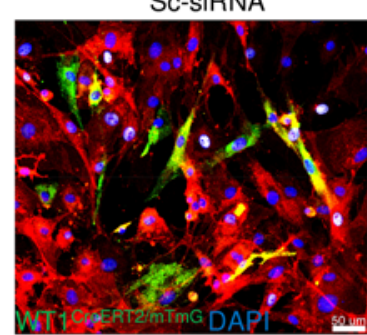

TGFa/WT1 ${ }^{+/-}$

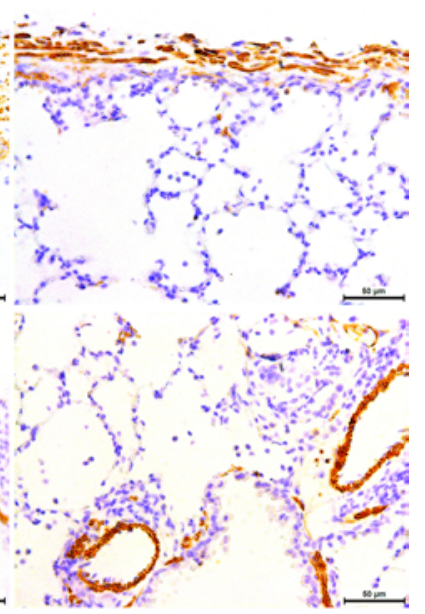

WT1-siRNA

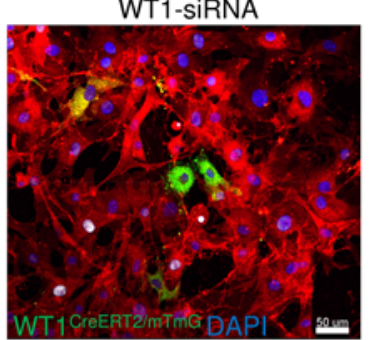

E

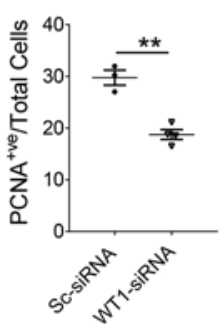

F

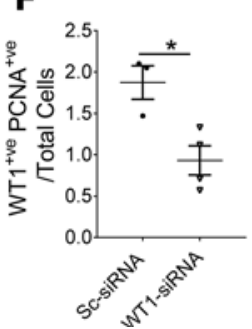

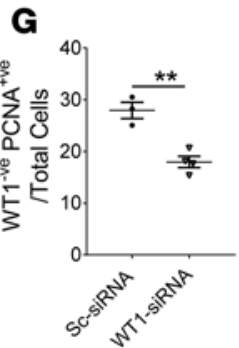

H

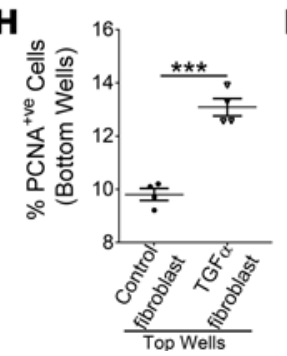

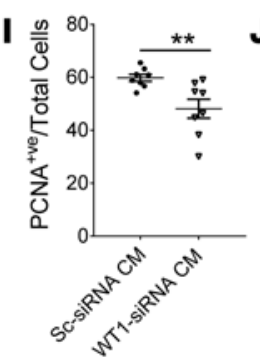

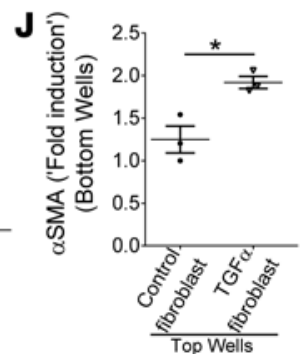

Figure 6. Mechanisms of WT1-driven fibroproliferation and myofibroblast transformation in vivo. (A) Immunostainings show Ki67-positive cells residing in subpleura and adventitia were reduced in TCF $\alpha / \mathrm{WT}^{+/-}$mice compared with TCF $\alpha / \mathrm{WT}^{+/+}$mice on Dox for 4 weeks. Scale bar: $50 \mu \mathrm{m}$. (B) Immunostainings show $\alpha$ SMA-positive cells residing in subpleura and adventitia were reduced in TCF $\alpha / \mathrm{WT}^{+/-}$mice compared with TCF $\alpha / \mathrm{WT} 1^{+/+}$mice on Dox for 4 weeks. Scale bar: $50 \mu \mathrm{m}$. (C) Immunostainings show $\alpha \mathrm{SMA}$-positive cells residing in subpleura and adventitia were reduced in WT1 ${ }^{+/-}$mice compared with WT1 ${ }^{+/+}$mice treated with bleomycin for 4 weeks. Scale bar: $200 \mu \mathrm{m}$. (D) Primary lung resident fibroblasts were isolated TCF $\alpha /$ WT1 1 reeRT2/mTm mice on Dox for 4 weeks and treated with either control or WT1 siRNA in presence of 4-hydroxy tamoxifen for 72 hours. Cells were immunostained for PCNA and immunofluorescence images were collected at original magnification $\times 10$ (Scale bar: $50 \mu \mathrm{m}$ ). (E-C) The number of PCNA-positive cells that coexpresses WT1 (green) in total DAPI-positive cells

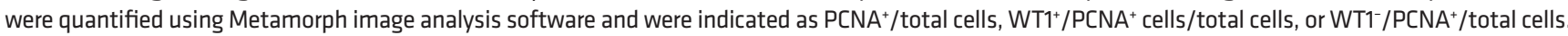
(H) Fibroblast cocultures experiments were performed using lung-resident fibroblasts isolated from control mice (bottom chamber) or TCF $\alpha$ transgenic mice on Dox for 6 weeks (top chambers). Cells in the bottom chambers were immunostained for PCNA. Images were collected at original magnification $\times 10$. Total DAPI-positive and PCNA-positive cells were quantified using ND2 analysis software. (I) Control mice fibroblasts were cultured for 48 hours in the presence of conditioned media obtained from control or WT1 siRNA transfected fibrotic fibroblasts for 72 hours. After 48 hours, cells were immunostained for PCNA. Images were collected at original magnification $\times 10$. Total DAPI-positive and PCNA-positive cells were quantified using ND2 analysis software. (J) Fibroblast cocultures experiments were performed using lung-resident fibroblasts isolated from the lung cultures of control $\alpha \mathrm{SMA} \mathrm{AreERT}^{\mathrm{m} / \mathrm{mTC}}$ mice (bottom chamber) or TCF $\alpha$ transgenic mice on Dox for 6 weeks (top chambers). RNA was isolated from the cell lysates of bottom chamber, and quantification of $\alpha$ SMA gene expression was performed. All data are representative of 2 independent experiments with similar results ( $n=3-4$ /group). All data in the figure are presented as mean \pm SEM. Statistical significance was calculated using unpaired 2-tailed Student's $t$ test for comparison between 2 groups. ${ }^{*} P<0.05,{ }^{* *} P<0.005$, ${ }^{* * *} P<0.0005$.

alter the proliferation of WT1-negative fibroblasts in a non-cell autonomous manner through secretion of paracrine factors. Using transwell coculture studies, the effect of the WT1-overexpressing fibroblasts on the myofibroblast transformation was determined by quantifying the transcripts of aSMA gene expression in the bottom chamber after 48 hours of coculture. We observed a significant increase in $\alpha$ SMA gene expression in the bottom chambers of cocultures with WT1-overexpressing fibroblasts compared with cocultures with WT1-negative fibroblasts from normal lungs (Figure 6J). These findings show 
that WT-expressing cells can induce fibroblast-to-myofibroblast transformation in a non-cell autonomous manner. Together, our findings suggest that WT1 can augment the proliferation and myofibroblast transformation in a non-cell autonomous manner through secretion of paracrine factors.

\section{Discussion}

Our study demonstrates a previously unknown role for WT1 as a positive regulator of pulmonary fibrosis. WT1 was detected in mesothelial and mesenchymal cells, but not epithelial cells, in lungs from patients with IPF. Using in vitro studies with isolated lung fibroblasts from a mouse model of pulmonary fibrosis and in vivo studies of developing pulmonary fibrosis, we demonstrate that WT1 functions to enhance proliferation, myofibroblast transformation, and ECM production. Haploinsufficiency of WT1 reduces fibroblast activation and pulmonary fibrosis in mouse models. WT1 acts by binding the SMA promoter, a marker of myofibroblasts. Using in vivo cell marking in mouse models, we identified WT1 as a key driver of mesothelial-to-myofibroblast and fibroblast-to-myofibroblast transformation. Our study demonstrates the developmental profile of WT1 expression. WT1 is coexpressed with calretinin, indicating expression in mesothelial cells during lung development but no expression in myofibroblasts or other cells in the lung parenchyma at E15.5, during embryonic stages of lung development. This increased WT1 expression in mesothelial cells is significantly downregulated at later prenatal or postnatal stages of lung development in mice. Importantly, our findings demonstrate that a limited number of mesothelial cells express WT1 in adult lungs compared with embryonic stages of lung development $(13,22,24)$. These findings are consistent with other published studies on WT1 expression in embryonic and adult lungs $(13,14)$. In contrast, we observed a robust increase in WT1 staining in both mesenchymal cells and mesothelial cells during TGF $\alpha$ - and bleomycin-induced pulmonary fibrosis in postnatal lungs (20). Localization of WT1 in the mouse models resembles localization of WT1 in subpleural regions of fibrotic lesions in IPF. The cellular localization of WT1 in either mesothelial cells or mesenchymal cells could reflect an early event in fibroblast activation rather than a simple association in the progressive expansion of fibrotic lesions $(22,39)$. Our findings support a pathogenic role for WT1 in fibroblast activation. Inhibition of WT1 caused a marked reduction in IPF-specific ECM genes such as Col14, Col15, Itg2, Itg7, and Lum and proliferative genes such as Grem1, Runx1, Wnt4, Stat3, Prrx1, Igf1, $C c n b 1$, and E2f8. These findings are consistent with previous studies in fibroblasts isolated from normal and IPF lungs that revealed that activation of Stat 3 by IL- 6 was mitogenic for IPF fibroblasts due to activation of cyclin D (40-42). Our data show that WT1 affects fibroproliferation in a non-cell autonomous manner, as the loss of WT1 in WT1-positive fibroblasts attenuated the proliferation in both WT1-positive and WT1negative fibroblasts. In contrast, overexpression of WT1 leads to a marked increase in the proliferation of human and mouse fibroblasts. In vivo, WT1 haploinsufficiency attenuated the proliferation of lung cells in both subpleural and adventitial areas of the lung. Reduced expression of WT1 attenuates the extent of fibrosis by modulating proliferation and ECM production. Taken together, these data highlight the complexity in the WT1 regulation of fibroproliferation and ECM production. Future experiments will need to address how WT1 influences the intracellular signaling and key gene networks in multiple cell types that can affect fibroproliferation and ECM production in the pathogenesis of pulmonary fibrosis.

Our lineage-tracing studies indicate that club-shaped mesothelial cells labeled by WT1 undergo proliferative expansion and differentiate into spindle-shaped myofibroblasts during TGF $\alpha$-induced pulmonary fibrosis in vivo. It has been reported that, during embryonic lung development, mesenchymal cells develop from pleural mesothelial cells, and recent studies identify a role for mesothelial cells as myofibroblast progenitors in the lung $(14,23,43)$, kidney (44-47), and liver $(48,49)$. However, a recent study by Karki and colleagues suggests that WT1 functions as a negative regulator of mesothelial-to-mesenchymal transition and WT1-derived mesenchymal cells migrate into the parenchyma within 4 hours of TGF $\beta$ treatment (23). In contrast, we and others have observed few WT1-positive mesothelial cells on the pleural surfaces of normal adult mice $(13,22,24)$, and WT1-positive mesothelial cells of adult mice contribute to myofibroblasts in subpleural fibrotic lesions, albeit in small numbers. A possible explanation for the above differences could be due to intranasal TGF $\beta$ causing different signaling or an insufficient dose that does not show WT1 effects compared with intradermal bleomycin- or TGF $\alpha$-induced pulmonary fibrosis. In support, our published studies have demonstrated that TGF $\alpha$-induced fibrosis is not altered by blocking $\alpha \mathrm{V} \beta 6 / \mathrm{TGF} \beta$ signaling (50). Findings in human IPF show that WT1 expression occurs in mesothelial cells, fibroblasts, and myofibroblasts of human IPF lungs but has limited or no expression in healthy human lungs (22). Additionally, our cell-tracking studies identified aSMA staining in cells that originate from WT1-positive mesothelial 
cells during TGF $\alpha$ - induced pulmonary fibrosis. A recent study by Boren and colleagues showed increases in the pleural mesothelial cells in patients diagnosed with nonspecific pleuritis, as well as mice intrapleurally injected with $S$. pneumoniae to induce pleural injury (51). Nonetheless, we do not know whether early upregulation of WT1 is limited to mesothelial cells alone or both mesothelial cells and mesenchymal cells contributing to myofibroblast transformation that occurs in subpleural fibrotic lesions. Notably, we show that there are more WT1-expressing mesenchymal cells in the subpleural regions of TGF $\alpha$ transgenic mice with continuous tamoxifen treatment compared with mice pretreated with tamoxifen. These observations imply that WT1 upregulation in both mesothelium and mesenchymal cells contribute to subpleural fibrotic lesions in pulmonary fibrosis. With continuous tamoxifen treatment, we found that the majority of mesenchymal cells in subpleura originate from WT1-positive progenitors. Future studies are needed to further delineate mechanisms whereby WT1 leads to differentiation of myofibroblasts in the progressive expansion of subpleural fibrotic lesions. Finally, our results imply that the WT1 upregulation, but not downregulation, is involved in mesothelial-to-myofibroblast transition in the development of pulmonary fibrosis.

Our data show that WT1 functions in a previously unknown manner as a positive regulator of myofibroblast differentiation via 2 potentially novel mechanisms. First, the $\alpha$ SMA gene is the direct target of transcriptional regulation by WT1, and the binding of WT1 induces $\alpha$ SMA transcripts, thus establishing a functional link to myofibroblast accumulation in subpleural areas of IPF lungs. Notably, WT1 binding elements in the aSMA gene are highly conserved among mammals, including mice and humans. Second, WT1-positive cells can induce fibroblast-to-myofibroblast transformation in a non-cell autonomous manner. Data from our coculture studies indicate a significant increase in fibroblast-to-myofibroblast transformation by WT1-overexpressing fibroblasts isolated from TGF $\alpha$ transgenic mice compared with normal fibroblasts that express limited or no WT1. Moreover, the marked upregulation of $\alpha$ SMA transcripts and increased myofibroblast accumulation after WT1 overexpression are consistent with the identification of a significant number of fibroblasts or myofibroblasts that coexpress WT1 in IPF lungs and mouse models of pulmonary fibrosis $(22,24)$. Furthermore, loss of WT1 attenuated myofibroblast accumulation in both subpleural and adventitial fibrotic lesions in vivo. Considered together, the data indicate that WT1 expression is a critical pathogenic event in myofibroblast transformation and ECM production. In support, we observed a strong correlation between collagen deposition and WT1 levels during TGF $\alpha$-induced pulmonary fibrosis (22).

Progressive thickening of subpleural and peripheral areas of the lung is a characteristic feature of IPF. Fibrosis in subpleural regions is also a predominant phenotype in other fibrotic lung diseases, including asbestos-related pleural fibrosis, collagen vascular disease resulting in interstitial fibrosis, and drug reactions with pleural involvement $(34,52,53)$. A prevailing view has been that lung-resident fibroblasts of subpleural fibrotic lesions invade into lung parenchyma to accumulate in peribronchial fibrotic lesions - a view supported indirectly by the observation that lung-resident fibroblasts are invasive (54-56). Our new findings support a model in which a mesothelial-to-fibroblast transformation is a local event that is confined to the subpleural area of the lung - a view supported by the lack of mesothelium-derived fibroblasts in peribronchial and alveolar areas of lungs of TGF $\alpha$ mice continuously treated with tamoxifen for 4 weeks, while on Dox. However, we find that WT1 expression in subpleural lesions is critical to the development of fibrotic lesions in other areas of the lung, including subpleural, peribronchial, and alveolar regions. By regulating the expression of distinct paracrine factors that may originate in subpleural regions, WT1 can function as a key driver to maintain pulmonary fibrosis in other peripheral areas of the lung. Our coculture studies further imply that no cell-to-cell contact is needed to initiate fibroblast-to-myofibroblast transformation in quiescent fibroblasts by WT1. Our in vivo studies demonstrated that the reduced expression of WT1 in subpleural regions of the lung attenuated both fibroproliferation and myofibroblast accumulation in the parenchymal areas of the lung. While it remains possible that a small amount of WT1 below the threshold of detection is present in lung parenchyma, it is unlikely to account for the large fibrotic phenotypes documented here. However, the identity of fibroblasts in lung parenchyma remains to be determined during TGF $\alpha$-induced pulmonary fibrosis. More recent findings have suggested that multiple mesenchymal cell lineages exist in distinct anatomical regions of the lung in promoting self-renewal versus a pathological change in injury $(35,38,56-59)$. While mesenchymal cells expressing Lgr5 were found mainly in the alveolar area of the lungs, Lgr6-positive mesenchymal cells can be found predominantly in peribronchiolar and adventitial regions of the lung (60). Our findings suggest WT1-expressing mesenchymal cells accumulate in subpleural areas of fibrotic lungs, but none are found in healthy lungs. Thus, within the lung, there appears to be a remarkable heterogeneity in mesenchymal cells and compartmentalization of effector signals that control 


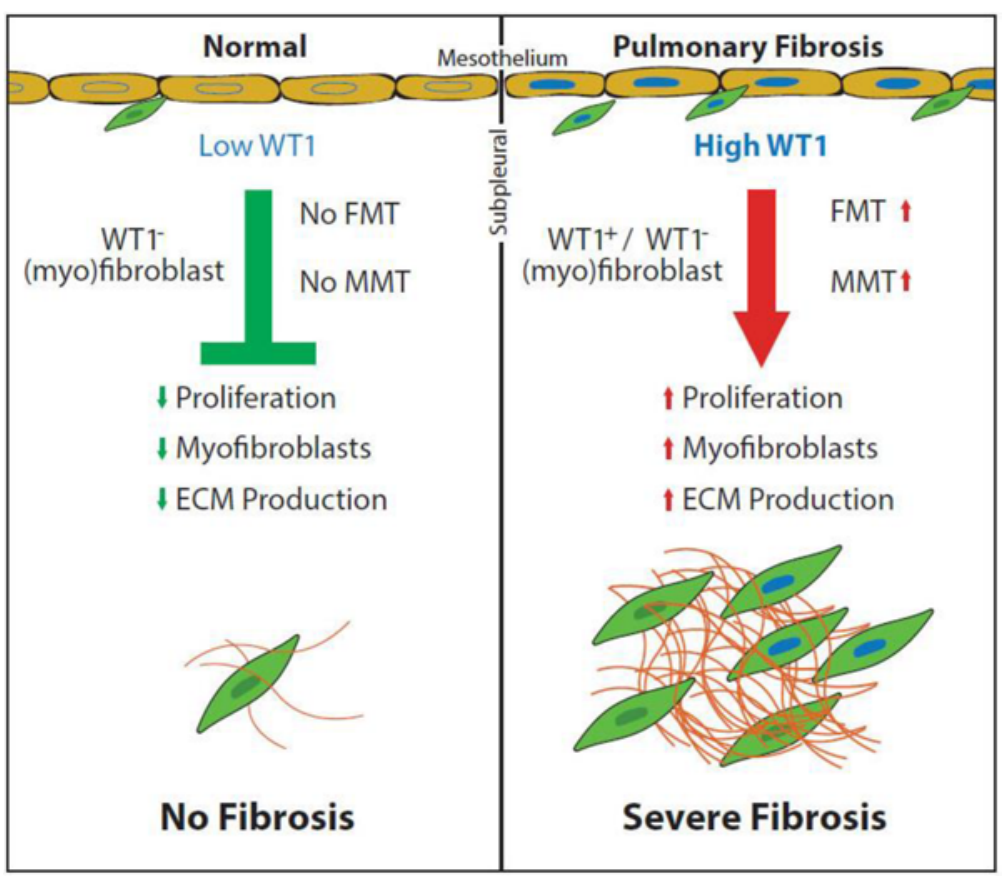

Figure 7. Schematic representation of WT1-driven fibroblast activation in pulmonary fibrosis. In the peripheral areas of the lung, WT1-positive mesothelial cells and mesenchymal cells undergo myofibroblast transformation and activate lung-resident fibroblasts via direct and indirect mechanisms in the pathogenesis of pulmonary fibrosis.

pulmonary fibrosis. Future studies are needed to investigate a possible cross-talk between WT1-positive cells of subpleural regions, with distinct populations documented in multiple areas of the lung. Our data indicate that future pharmacologic interventions targeting WT1-driven events in subpleural fibrotic machinery could target profibrotic processes of the lung in a global and a coordinated fashion.

In summary, our data provides mechanistic insights into the molecular cause of subpleural thickening in IPF by revealing a role of WT1 and targets that are either directly or indirectly involved in fibroblastspecific functions and processes (Figure 7). The largest category of genes altered by WT1 are known to regulate proliferation, growth, differentiation, and ECM production. Concurrently, using 2 different mouse models of pulmonary fibrosis, we have demonstrated that the upregulation of WT1 in subpleural fibrotic lesions causes severe fibrotic lung disease. The findings of this study identify a previously unknown role for WT1 in pathological fibroblast activity in IPF, through acceleration of fibroproliferation and myofibroblast transformation through direct and indirect mechanisms. Overall, our results suggest that WT1 may be a therapeutic target in modulating pulmonary fibrosis.

\section{Methods}

Mouse strains. Generation of WT1 ${ }^{\text {CreERT2/- }}$ transgenic mice has been described previously (16). Heterozygous WT1 ${ }^{\text {CreERT2/- }}$ knock-in mice are haploinsufficient for the WT1 allele (WT1 ${ }^{+/-}$) and display no developmental abnormalities. Clara cell-specific promoter-driven (CCSP-driven) TGF $\alpha$-overexpression mice were generated as described previously (61). We performed sequential breeding of CCSP/TGF $\alpha$ transgenic mice with WT1 $1^{\text {CreERT2/- }}$ mice to generate control or TGF $\alpha$ transgenic mice with WT (WT1 $1^{+/+}$ and $\mathrm{TGF} \alpha / \mathrm{WT}^{+/+}$) or WT1 mutant allele $\left(\mathrm{WT} 1^{+/-}\right.$and $\left.\mathrm{TGF} \alpha / \mathrm{WT}^{+/-}\right)$. The generated $\mathrm{WT} 1^{\text {CreERT2/mTm }}$ reporter mice were described previously (22). TGF $\alpha / \mathrm{WT} 11^{C r E R T 2 / \mathrm{mTmG}}$ quadruple transgenic mice were generated by crossing the CCSP/TGF $\alpha$ mice with $\mathrm{WT} 11^{\text {CreERT2/mTmG }}$ reporter mice. Ten- to 14 -week-old mice were used in all experiments. $\alpha \mathrm{SMA}^{Y F P}$ reporter mice used in the confocal analysis of WT1 expression in embryonic lung development and transcript analysis were described previously and used to efficiently label embryonic and adult smooth muscle cells $(62,63)$. The heterozygous $\alpha \mathrm{SMA}^{\text {CreERT2/mTmG }}$ male transgenic mouse strain was generated as previously described (30). These mice express CreERT2 under the control of the promoter for mouse smooth muscle myosin, heavy polypeptide 11 and have been shown to track the genetic lineage of $\alpha$-SMA-expressing cells (64). 
Mouse models of TGF $\alpha$ - and bleomycin-induced pulmonary fibrosis. TGF $\alpha$ - overexpression was induced by administering food containing Dox $(62.5 \mathrm{mg} / \mathrm{kg}$ ) (catalog D9891, MilliporeSigma) to the CCSP/TGF $\alpha$ transgenic mice. CCSP/- littermate mice fed with Dox-treated food were used as controls (55). For the mouse model of bleomycin-induced pulmonary fibrosis, 10- to 14-week-old mice received bleomycin intradermally (6 U per kg bodyweight/day) suspended in $50 \mu 1$ saline solution in the center of the shaved back (within a 50-mm radius) for 5 days per week for a total of 4 weeks. At day 28, mice were euthanized and lung samples were collected for further analysis.

Tamoxifen treatments. Tamoxifen (catalog T5648, MilliporeSigma) was dissolved in ethanol and emulsified in sunflower oil (catalog W530285, MilliporeSigma) at $25 \mathrm{mg} / \mathrm{ml}$ concentration as described (22), and mice were treated with tamoxifen (i.p. $2.5 \mathrm{mg}$ /day) to induce Cre recombination via i.p. injections.

Histology and IHC. Formalin- or OCT-fixed mouse lung tissue sections were prepared and stained with Masson's trichrome or immunostained with antibodies against $\alpha$-SMA or Ki67 as described previously (65).

Human and mouse primary lung fibroblast cultures. Human and mouse primary lung fibroblast cell cultures were prepared as previously described $(55,65)$.

SiRNA transfections. Primary human or mouse fibroblast cells were transfected with stealth siRNA (human WT1 siRNA [catalog HSS111390, Invitrogen], mouse WT1 siRNA [catalog MSS212628, Invitrogen], or stealth negative control siRNA [catalog 12935300, Invitrogen]) using the Lipofectamine 3000 Transfection kit (catalog L3000-015, Invitrogen) as previously described (30). Transfected cells were harvested 72 hours after transfection and used for RNA isolation and whole transcriptome analysis.

Whole-transcriptome shotgun sequencing (RNA-Seq) and bioinformatic analysis. Total RNA was prepared from primary lung fibroblasts isolated from lung cultures of TGF $\alpha$ mice on Dox food for 10 days and transfected with either control or WT1 siRNA as described earlier (22). RNA-Seq was performed using an Illumina HiSeq-1000 Sequencer as described previously (66). Complete RNA-Seq data are available at a gene expression omnibus or GEO database (GEO accession number GSE110177). A previously published transcriptomic data set (GSE53845) derived from analysis of the lung biopsies of 40 IPF patients and 8 healthy controls available in the National Center for Biotechnology Information (NCBI) GEO was used to identify differentially expressed genes (8). Differential analysis for genes was performed using the $\mathrm{R}$ package 'limma' or GEO2R (http://www.ncbi.nlm.nih.gov/geo/geo2r/) with $P$ value and FDR threshold at 0.05 and fold-change threshold at 1.5. This IPF gene signature was queried against the WT1 siRNAgenerated gene expression signature. Functional enrichment analysis of the negatively correlated gene sets between IPF lungs and WT1 siRNA-treated cells were analyzed using the ToppFun application of the ToppGene Suite (31). For network representation of select significantly enriched biological processes and pathways, we used Cytoscape (67).

Viral transductions. Primary lung-resident fibroblasts were isolated from the lung cultures of human or mouse lungs by negative selection for anti-CD45-stained cells using MACS columns (68). Thus, isolated fibroblasts were transduced with control or WT1-overexpressing adenovirus (human WT1) (catalog 000488A, Abm) and lentivirus (mouse WT1) (catalog 41082, Addgene) (69) or Control lentivirus (plasmid lentivirus, catalog 8453, Addgene) (1-10 MOI) for 48-72 hours.

Myofibroblast differentiation. In vitro fibroblast-to-myofibroblast transformation assay was performed using primary lung-resident fibroblasts isolated from the lung cultures of $\alpha \mathrm{SMA}^{\mathrm{CrEERT2} / \mathrm{mTmG}}$ mice as previously described (30). Fibroblasts were seeded onto chamber slides and serum starved ( $0.5 \%$ FBS) at $70 \%$ $80 \%$ confluency overnight and then treated with control or WT1-overexpressing lentivirus in the presence of $2 \mu \mathrm{M}$ (Z)-4-hydroxytamoxifen (catalog H7904, MilliporeSigma) for 72 hours. For WT1 knockdown studies, isolated lung-resident fibroblasts were transfected with either control or WT1-specific siRNA for 24 hours; after transfection, cells were treated with $2 \mu \mathrm{M}$ 4-hydroxytamoxifen for an additional 48 hours. After 72 hours, cells were fixed with 4\% paraformaldehyde, and the nucleus was stained using ProLong gold DAPI (Thermo Fisher Scientific). Fluorescence images were collected using a Nikon AIR-A1 laserscanning confocal microscope. Cell quantitation was performed using Metamorph imaging software.

Proliferation assays. Primary lung-resident fibroblast proliferation was examined using BrdU (catalog 6813S, Cell Signaling Technology) incorporation as described previously (30).

Confocal imaging. Mouse lungs were embedded in OCT medium, and sections were prepared as described previously (30). Lungs were immunostained against WT1, calretinin, or aSMA antigens. Appropriate secondary antibodies conjugated with Alexa Fluor 488, Alexa Fluor 594, or Alexa Fluor 647 (Invitrogen), respectively, were used for visualization (antibody details in supplemental Table 4). 
For PCNA immunostainings, cells were fixed in ice cold methanol. Nonspecific protein binding was blocked with M.O.M. blocking solution (catalog BMK2202, Vector Laboratories) in PBS, followed by incubation with primary antibodies in M.O.M. diluent solution and subsequent incubation with secondary antibodies. Finally, chamber slides were washed and mounted with ProLong gold DAPI. Confocal images were collected using a Nikon AIR-A1 laser-scanning confocal microscope. Imaris (version 7.2.0; Bitplane) was used for image analysis. For quantitative analysis of PCNA, 5-7 random images were taken for each condition (with the $10 \times$ objective). Cell quantitation was performed using Metamorph imaging software.

Chip-Seq PCR assay. ChIP was performed using the ChIP assay kit (catalog 9005, Cell Signaling Technology). Briefly, IMR-90 fibroblasts were treated with WT1-overexpressing adenovirus (10 MOI) for 48 hours. Cells were cross-linked with 1\% formaldehyde, and immunoprecipitation was performed with anti-WT1 (catalog 12609-1-AP, Proteintech) or isotype control IgG (catalog 2729, Cell Signaling Technology) at $4^{\circ} \mathrm{C}$ as described in the manufacturer's protocol. DNA was purified, and PCR was performed in $25 \mu \mathrm{l}$ of PCR-reaction mix using $1 \mu \mathrm{l}$ of DNA in a Veriti Thermal Cycler (Applied Biosystems). The primer sequences, annealing temperatures, and PCR product sizes are provided in Supplemental Table 1. The PCR product was electrophoresed through a $2.0 \%$ agarose gel with ethidium bromide using $1 \times$ TAE buffer (Thermo Fisher Scientific), and resulting fragments were visualized using a UV transilluminator (FotoDyne).

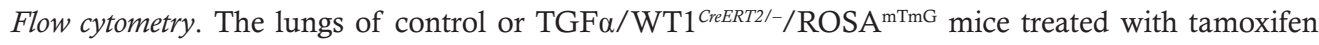
and Dox were cultured for 7 days, and the total lung fibroblasts were used for flow cytometry analysis. Data were acquired using LSR Fortessa flow cytometer (BD Biosciences). Unstained and single-stained (tdTomato or EGFP) total lung cells were used for compensation and gating. Data were analyzed using FACSDIVA software (BD Biosciences).

$R N A$ preparation and $R T-P C R$. Total RNA was extracted using the RNAeasy Mini Kit (Qiagen Sciences) as previously described (22). cDNA synthesis was carried out using SuperScript III Reverse Transcriptase (Invitrogen). Quantitative PCR (qPCR) assays were performed with the CFX384 Touch Real-Time PCR Detection system (Bio-Rad). The relative quantities of mRNA for several genes were determined using iTaq universal SYBR green supermix (Bio-Rad). Target-gene transcripts in each sample were normalized to hypoxanthine guanine phosphoribosyl transferase or HPRT or $\beta$-actin and expressed as a relative increase or decrease compared with control. All real-time primer sequences used are shown in Supplemental Tables 2 and 3 .

Western blot. Purified lung-resident fibroblasts were isolated as described earlier (22) and cultured on 12 -well plates to $90 \%$ confluence and transduced with control Adenovirus or WT1 adenovirus for 72 hours. Cells were lysed in RIPA lysis buffer, and protein estimation was performed using the BCA method according to manufactures instructions (catalog 23225, Thermo Fisher Scientific). Immunoblotting and quantification were performed using the volume-integration function of Phosphor Imager Software Image Quant 5.2, as previously described (22). Primary antibodies and dilutions used are described in Supplemental Table 5, and the appropriate secondary antibodies conjugated with peroxidase (1:1000) were used for blot development.

Statistics. All data were analyzed using Prism (Version 7; Graph Pad). One-way ANOVA with Sidak's multiple comparison post-test was used to compare different experimental groups. Student's two-tailed $t$ test was used to compare between 2 experimental groups. Data were considered statistically significant for $\mathrm{P}$ values less than 0.05 .

Study approval. All mice were housed under specific pathogen-free conditions, and all animal experimental protocols were approved by the Institutional Animal Care and Use Committee of Cincinnati Children's Hospital Medical Center.

\section{Author contributions}

SKM and VS conceived the project, designed experiments, analyzed data, and wrote the manuscript; VS performed most of the experiments. AGJ performed bioinformatic analysis and provided suggestions; RKK performed immunostainings; ESW provided human lung tissues, IPF cells, and RNA; DS provided embryonic mouse lungs and $\alpha$ SMA reporter mice; GBR provided analytical tools and suggestions; and TRK assisted in transgenic mice generation and edited the manuscript. 


\section{Acknowledgments}

The authors thank the veterinary services, flow cytometry core, and pathology research core at Cincinnati Children's Hospital Medical Center for the help in this study. We thank Frank McCormack and Jeffrey Whitsett for valuable suggestions and Gail Pyne-Geithman for editorial assistance. This research was supported by the NIH grants NHLBI 1R21HL133539 (SKM and AGJ) and 1R01 HL134801 (SKM), the US Department of Defense funds, W81XWH-17-1-0666 (SKM), NHLBI R03HL133420 (DS), and Department of Biotechnology Fellowship, Government of India (RKK).

Address correspondence to: Satish K. Madala, Division of Pulmonary Medicine, Cincinnati Children's Hospital Medical Center, MLC 2021, 3333 Burnet Avenue, Cincinnati, Ohio 45229, USA. Phone: 513.636.9852; Email: satish.madala@cchmc.org.

1. Raghu G, Weycker D, Edelsberg J, Bradford WZ, Oster G. Incidence and prevalence of idiopathic pulmonary fibrosis. Am J Respir Crit Care Med. 2006;174(7):810-816.

2. Wynn TA. Cellular and molecular mechanisms of fibrosis. J Pathol. 2008;214(2):199-210.

3. Ley B, Collard HR. Epidemiology of idiopathic pulmonary fibrosis. Clin Epidemiol. 2013;5:483-492.

4. Nalysnyk L, Cid-Ruzafa J, Rotella P, Esser D. Incidence and prevalence of idiopathic pulmonary fibrosis: review of the literature. Eur Respir Rev. 2012;21(126):355-361.

5. Raghu G, et al. An official ATS/ERS/JRS/ALAT statement: idiopathic pulmonary fibrosis: evidence-based guidelines for diagnosis and management. Am J Respir Crit Care Med. 2011;183(6):788-824.

6. Smith ML. Update on Pulmonary Fibrosis: Not All Fibrosis Is Created Equally. Arch Pathol Lab Med. 2016;140(3):221-229.

7. Cool CD, Groshong SD, Rai PR, Henson PM, Stewart JS, Brown KK. Fibroblast foci are not discrete sites of lung injury or repair: the fibroblast reticulum. Am J Respir Crit Care Med. 2006;174(6):654-658.

8. DePianto DJ, et al. Heterogeneous gene expression signatures correspond to distinct lung pathologies and biomarkers of disease severity in idiopathic pulmonary fibrosis. Thorax. 2015;70(1):48-56.

9. Chau YY, Hastie ND. The role of Wt1 in regulating mesenchyme in cancer, development, and tissue homeostasis. Trends Genet. 2012;28(10):515-524.

10. Bansal H, et al. Heat shock protein 90 regulates the expression of Wilms tumor 1 protein in myeloid leukemias. Blood. 2010;116(22):4591-4599.

11. Rampal R, Figueroa ME. Wilms tumor 1 mutations in the pathogenesis of acute myeloid leukemia. Haematologica. 2016;101(6):672-679.

12. Duim SN, Goumans MJ, Kruithof BPT. Chapter 13 WT1 in Cardiac Development and Disease. In: van den Heuvel-Eibrink MM ed. Wilms Tumor. Brisbane (AU);Codon Publications:2016. https://www.ncbi.nlm.nih.gov/books/NBK373348/doi: 10.15586/codon.wt.2016.ch13. Accessed August 1, 2018.

13. Que J, Wilm B, Hasegawa H, Wang F, Bader D, Hogan BL. Mesothelium contributes to vascular smooth muscle and mesenchyme during lung development. Proc Natl Acad Sci USA. 2008;105(43):16626-16630.

14. Moiseenko A, et al. Origin and characterization of alpha smooth muscle actin-positive cells during murine lung development. Stem Cells. 2017;35(6):1566-1578.

15. Hohenstein P, Pritchard-Jones K, Charlton J. The yin and yang of kidney development and Wilms' tumors. Genes Dev. 2015;29(5):467-482.

16. Zhou B, et al. Epicardial progenitors contribute to the cardiomyocyte lineage in the developing heart. Nature. 2008;454(7200):109-113.

17. Gebeshuber CA, et al. Focal segmental glomerulosclerosis is induced by microRNA-193a and its downregulation of WT1. Nat Med. 2013;19(4):481-7.

18. Bandiera R, Vidal VP, Motamedi FJ, Clarkson M, Sahut-Barnola I, von Gise A, et al. WT1 maintains adrenal-gonadal primordium identity and marks a population of AGP-like progenitors within the adrenal gland. Developmental Cell. 2013;27(1):5-18.

19. Shandilya J, Roberts SG. A role of WT1 in cell division and genomic stability. Cell Cycle. 2015;14(9):1358-1364.

20. Shandilya J, Toska E, Richard DJ, Medler KF, Roberts SG. WT1 interacts with MAD2 and regulates mitotic checkpoint function. Nat Commun. 2014; 5:4903.

21. Vicent S, et al. Wilms tumor 1 (WT1) regulates KRAS-driven oncogenesis and senescence in mouse and human models. $J$ Clin Invest. 2010;120(11):3940-3952.

22. Sontake V, et al. Fibrocytes Regulate Wilms Tumor 1-Positive Cell Accumulation in Severe Fibrotic Lung Disease. J Immunol. 2015;195(8):3978-3991.

23. Karki S, et al. Wilms' tumor 1 (Wt1) regulates pleural mesothelial cell plasticity and transition into myofibroblasts in idiopathic pulmonary fibrosis. FASEB J. 2014;28(3):1122-1131.

24. von Gise A, et al. Contribution of Fetal, but Not Adult, Pulmonary Mesothelium to Mesenchymal Lineages in Lung Homeostasis and Fibrosis. Am J Respir Cell Mol Biol. 2016;54(2):222-230.

25. Luzina IG, et al. Transcriptomic evidence of immune activation in macroscopically normal-appearing and scarred lung tissues in idiopathic pulmonary fibrosis. Cell Immunol. 2018;325:1-13.

26. Scotton CJ, Chambers RC. Molecular targets in pulmonary fibrosis: the myofibroblast in focus. Chest. 2007;132(4):1311-1321.

27. Phan SH. The myofibroblast in pulmonary fibrosis. Chest. 2002;122(6 Suppl):286S-289S.

28. Hinz B, Phan SH, Thannickal VJ, Galli A, Bochaton-Piallat ML, Gabbiani G. The myofibroblast: one function, multiple origins. Am J Pathol. 2007;170(6):1807-1816.

29. Klingberg F, Hinz B, White ES. The myofibroblast matrix: implications for tissue repair and fibrosis. J Pathol. 2013;229(2):298-309. 
30. Sontake V, et al. Hsp90 regulation of fibroblast activation in pulmonary fibrosis. JCI Insight. 2017;2(4):e91454.

31. Chen J, Bardes EE, Aronow BJ, Jegga AG. ToppGene Suite for gene list enrichment analysis and candidate gene prioritization. Nucleic Acids Res. 2009;37(Web Server issue):W305-W311.

32. Hutchison N, Fligny C, Duffield JS. Resident mesenchymal cells and fibrosis. Biochim Biophys Acta. 2013;1832(7):962-971.

33. Phan SH. Fibroblast phenotypes in pulmonary fibrosis. Am J Respir Cell Mol Biol. 2003;29(3 Suppl):S87-S92.

34. Noble PW, Barkauskas CE, Jiang D. Pulmonary fibrosis: patterns and perpetrators. J Clin Invest. 2012;122(8):2756-2762.

35. Xia H, et al. Identification of a cell-of-origin for fibroblasts comprising the fibrotic reticulum in idiopathic pulmonary fibrosis. Am J Pathol. 2014;184(5):1369-1383.

36. Moore BB, Kolb M. Fibrocytes and progression of fibrotic lung disease. Ready for showtime? Am J Respir Crit Care Med. 2014;190(12):1338-1339.

37. Kleaveland KR, et al. Fibrocytes are not an essential source of type I collagen during lung fibrosis. J Immunol. 2014;193(10):5229-5239.

38. Hardie WD, et al. Signaling pathways in the epithelial origins of pulmonary fibrosis. Cell Cycle. 2010;9(14):2769-2776

39. Luzina IG, et al. Transcriptomic evidence of immune activation in macroscopically normal-appearing and scarred lung tissues in idiopathic pulmonary fibrosis. Cell Immunol. 2018;325:1-13.

40. Moodley YP, et al. Fibroblasts isolated from normal lungs and those with idiopathic pulmonary fibrosis differ in interleukin-6/ gp130-mediated cell signaling and proliferation. Am J Pathol. 2003;163(1):345-354

41. Pechkovsky DV, et al. STAT3-mediated signaling dysregulates lung fibroblast-myofibroblast activation and differentiation in UIP/IPF. Am J Pathol. 2012;180(4):1398-1412.

42. Pedroza M, et al. STAT-3 contributes to pulmonary fibrosis through epithelial injury and fibroblast-myofibroblast differentiation. FASEB J. 2016;30(1):129-140.

43. Zolak JS, et al. Pleural mesothelial cell differentiation and invasion in fibrogenic lung injury. Am J Pathol. 2013;182(4):1239-1247.

44. Kreidberg JA, et al. WT-1 is required for early kidney development. Cell. 1993;74(4):679-691.

45. Hartwig S, et al. Genomic characterization of Wilms' tumor suppressor 1 targets in nephron progenitor cells during kidney development. Development. 2010;137(7):1189-1203.

46. Kreidberg JA. WT1 and kidney progenitor cells. Organogenesis. 2010;6(2):61-70.

47. Motamedi FJ, et al. WT1 controls antagonistic FGF and BMP-pSMAD pathways in early renal progenitors. Nat Commun. 2014;5:4444.

48. Asahina K. Hepatic stellate cell progenitor cells. J Gastroenterol Hepatol. 2012;27 Suppl 2:80-84.

49. Ijpenberg A, et al. Wt1 and retinoic acid signaling are essential for stellate cell development and liver morphogenesis. Dev Biol. 2007;312(1):157-170.

50. Madala SK, et al. Inhibition of the $\alpha v \beta 6$ integrin leads to limited alteration of TGF- $\alpha$-induced pulmonary fibrosis. Am JPhysiol Lung Cell Mol Physiol. 2014;306(8):L726-L735.

51. Boren J, et al. Inhibition of Glycogen Synthase Kinase $3 \beta$ Blocks Mesomesenchymal Transition and Attenuates Streptococcus pneumonia-Mediated Pleural Injury in Mice. Am J Pathol. 2017;187(11):2461-2472.

52. Schwartz DA, Galvin JR, Yagla SJ, Speakman SB, Merchant JA, Hunninghake GW. Restrictive lung function and asbestosinduced pleural fibrosis. A quantitative approach. J Clin Invest. 1993;91(6):2685-2692.

53. Schneider F, Gruden J, Tazelaar HD, Leslie KO. Pleuropulmonary pathology in patients with rheumatic disease. Arch Pathol Lab Med. 2012;136(10):1242-1252.

54. Li Y, et al. Severe lung fibrosis requires an invasive fibroblast phenotype regulated by hyaluronan and CD44. J Exp Med. 2011;208(7):1459-1471.

55. Madala SK, Edukulla R, Schmidt S, Davidson C, Ikegami M, Hardie WD. Bone marrow-derived stromal cells are invasive and hyperproliferative and alter transforming growth factor- $\alpha$-induced pulmonary fibrosis. Am J Respir Cell Mol Biol. 2014;50(4):777-786.

56. Habiel DM, Hogaboam C. Heterogeneity in fibroblast proliferation and survival in idiopathic pulmonary fibrosis. Front Pharmacol. $2014 ; 5: 2$.

57. Rock JR, et al. Multiple stromal populations contribute to pulmonary fibrosis without evidence for epithelial to mesenchymal transition. Proc Natl Acad Sci USA. 2011;108(52):E1475-E1483.

58. O'Dwyer DN, Ashley SL, Moore BB. Influences of innate immunity, autophagy, and fibroblast activation in the pathogenesis of lung fibrosis. Am J Physiol Lung Cell Mol Physiol. 2016;311(3):L590-L601.

59. Zepp JA, et al. Distinct Mesenchymal Lineages and Niches Promote Epithelial Self-Renewal and Myofibrogenesis in the Lung. Cell. 2017;170(6):1134-1148.e10.

60. Lee JH, et al. Anatomically and Functionally Distinct Lung Mesenchymal Populations Marked by Lgr5 and Lgr6. Cell. 2017;170(6):1149-1163.e12.

61. Hardie WD, Le Cras TD, Jiang K, Tichelaar JW, Azhar M, Korfhagen TR. Conditional expression of transforming growth factor-alpha in adult mouse lung causes pulmonary fibrosis. Am J Physiol Lung Cell Mol Physiol. 2004;286(4):L741-L749.

62. Li J, Qu X, Bertram JF. Endothelial-myofibroblast transition contributes to the early development of diabetic renal interstitial fibrosis in streptozotocin-induced diabetic mice. Am J Pathol. 2009;175(4):1380-1388.

63. Cool J, Carmona FD, Szucsik JC, Capel B. Peritubular myoid cells are not the migrating population required for testis cord formation in the XY gonad. Sex Dev. 2008;2(3):128-133.

64. Wirth A, et al. G12-G13-LARG-mediated signaling in vascular smooth muscle is required for salt-induced hypertension. Nat Med. 2008;14(1):64-68.

65. Madala SK, Sontake V, Edukulla R, Davidson CR, Schmidt S, Hardie WD. Unique and Redundant Functions of p70 Ribosomal S6 Kinase Isoforms Regulate Mesenchymal Cell Proliferation and Migration in Pulmonary Fibrosis. Am J Respir Cell Mol Biol. 2016;55(6):792-803.

66. Madala SK, et al. Dual targeting of MEK and PI3K pathways attenuates established and progressive pulmonary fibrosis. PLoS ONE. 2014;9(1):e86536.

67. Shannon P, et al. Cytoscape: a software environment for integrated models of biomolecular interaction networks. Genome Res. 2003;13(11):2498-2504. 
68. Madala SK, Edukulla R, Schmidt S, Davidson C, Ikegami M, Hardie WD. Bone marrow-derived stromal cells are invasive and hyperproliferative and alter transforming growth factor-alpha-induced pulmonary fibrosis. Am J Respir Cell Mol Biol. 2014;50(4):777-86.

69. Buganim Y, et al. Direct reprogramming of fibroblasts into embryonic Sertoli-like cells by defined factors. Cell Stem Cell. 2012;11(3):373-386 